\title{
On the quantization of wave fields ${ }^{\star}, \star \star$
}

\author{
L. Rosenfeld ${ }^{\mathrm{a}}$ \\ Physics Institute of the Swiss Federal Institute of Technology, Zurich, Switzerland
}

Received 16 June 2016 / Received in final form 2 November 2016

Published online 11 January 2017

(C) EDP Sciences, Springer-Verlag 2017

\section{Introduction}

Heisenberg and Pauli ${ }^{1}$ have recently made substantial progress in the formulation of the general quantum mechanical laws for electromagnetic and material wave fields through the systematic development of Dirac's method of second quantization. In addition to certain deeper lying technical difficulties a characteristic difficulty of a more formal nature appeared; the momentum conjugate to the scalar potential vanishes identically. The formation of the Hamilton function and the commutation relations cannot be carried out without further work. Three methods have been proposed to date to resolve these problems. They do, to be sure, fulfill their objective but they can hardly be viewed as satisfactory.

1. The first Heisenberg-Pauli method is a purely analytical artifice ${ }^{2}$. Certain terms are added to the Lagrange function, multiplied by a small parameter $\epsilon$. These have the effect that the above-mentioned momentum no longer vanish. In the final result one then takes the limit $\epsilon=0$. However, the $\epsilon$-terms lead to unphysical caculational complications $^{3}$ and destroy the characteristic invariance of the Lagrangian under the gauge invariance group.

2. The second Heisenberg-Pauli method ${ }^{4}$, however, uses this invariance in an essential way. The scalar potential is given a certain arbitrary value, e.g. zero; then the Hamiltonian method delivers one less equation of motion. Supposing the missing equation of motion is $C=0$, then one finds as a consequence of the gauge invariance of the Hamiltonian that $C=$ constant. The choice of the value 0 for this constant

* Originally published in German "Zur Quantelung der Wellenfelder" in Annalen der Physik 397, 113 (1930). Submitted for publication on March 18, 1930

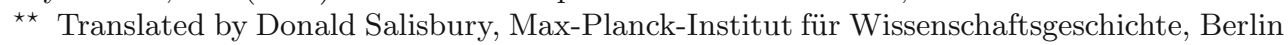
and Austin College, Sherman, TX, USA and Kurt Sundermeyer, Max-Planck-Institut für Wissenschaftsgeschichte, Berlin. e-mail: DSalisbury@austincollege.edu

a Deceased 23 March 1974.

1 W. Heisenberg, W. Pauli, Zeit. F. Phys. 54, 1 (1929); 59, 168 (1930). In the following referred to as H.P.I and H.P.II.

${ }^{2}$ H.P.I, pages 24-29, $30 \mathrm{ff}$.

3 Cf. L. Rosenfeld, Zeit. F. Phys. 58, 540 (1929).

${ }^{4}$ H.P.II. 
signifies a restriction to one of many distinct and independent systems of terms. But distinguishing a component of the 4-potential necessitates a proof of the relativistic covariance of this method, and this check is very cumbersome.

3. The Fermi method ${ }^{5}$ consists also in adding terms to the Lagrangian in such a manner that no momentum vanishes identically. In order that the resulting field equations agree with the usual equations certain constraints must be fulfilled; then it must be shown that when these constraints hold on a $t=$ constant slice they continue to hold under propagation in time. The disadvantage of this method is that once again the gauge invariance is destroyed.

The identical vanishing of the cited momentum component is by no means an isolated phenomenon; the origin is just the gauge invariance of the Lagrangian as is shown in detail below in a simple, comprehensive discussion. In an analogous fashion, i.e., more generally, the appearance of identical relations between variables and conjugate momenta is to be expected in all cases in which the Lagrangian permits a suitably built group. In investigating these relations in the especially instructive example of gravitation theory, Professor Pauli helpfully indicated to me a new method that allows one to construct a Hamiltonian procedure in a definitely simpler and natural way when identities are present, without being subjected to the disadvantages of the earlier methods. In the following the subject will be treated first from a general group theoretical perspective, and then illustrated with the help of physical examples ${ }^{6}$.

\section{Part one: General theory}

\subsection{Assumptions about the Lagrange function and the underlying group}

We consider any dynamical system defined through the field quantities $Q_{\alpha}\left(x^{1}, x^{2}, x^{3}, x^{4}\right)$ that depend on the spatial coordinates $x^{1}, x^{2}, x^{3}$ and the time coordinate $x^{4}=c t$ (and not, as in H. P., $x^{4}=i c t$ !). We need to make no assumptions about the Lagrange function $\mathcal{L}\left(Q ; \frac{\partial Q}{\partial x}\right)$ as long as we remain in the framework of the classical theory, i.e., we work only with $c$-numbers. If, however, we were to consider the $Q$ variables as q-numbers (while the spacetime coordinates always remain $c$-numbers) then we would have to take into account that the rule for the derivative of the function of a function would lose its general validity ${ }^{7}$. If we want to keep certain properties of the Lagrange function that follow from this rule (and this will be the case) we are forced to make certain restrictive assumptions about the function $\mathcal{L}$ such that these properties are valid in spite of the failure of the said derivative rule. It turns out that from the mathematical point of view these restrictions will have to be extensive, though for physically interesting Lagrangians they are fulfilled. They concern on the one hand the analytical nature of the Langrangian: It must be at most quadratic in the derivatives of the $Q_{\alpha}$, and furthermore, the ordering of mutually non-commutative quantities.

To abbreviate we often write $Q_{\alpha, \nu}$ instead of $\frac{\partial Q_{\alpha}}{\partial x^{\nu}}$, and also $\dot{Q}_{\alpha}$ instead of $Q_{\alpha, 4} \equiv$ $\frac{\partial Q_{\alpha}}{\partial x^{4}}$. Furthermore we will suppress the summation sign following the familiar rule. With these agreements our ansatz for the Lagrange function takes the form

$$
2 \mathcal{L}=Q_{\alpha, \nu} \mathcal{A}^{\alpha \nu, \beta \mu}(Q) Q_{\beta, \mu}+Q_{\alpha, \nu} \mathcal{B}^{\alpha \nu}(Q)+\mathcal{B}^{\alpha \nu}(Q) Q_{\alpha, \nu}+\mathcal{C}(Q) .
$$

${ }^{5}$ Cf. H.P.II, page 171 , footnote.

${ }^{6}$ Here I want to stress once and for all that in the special cases treated in the works H.P. I and H.P.II the path to the desired generalization frequently suggested itself to me. It would serve little purpose in the following to refer to each and every instance in which this occurred.

7 Cf. H.P.I, p. 18, further p. 14, footnote 1. 
Although only the $\dot{Q}_{\alpha}$ do not commute with the $Q_{\alpha}$, we must nevertheless retain a fixed factor ordering for the remaining derivatives since certain operations, $\frac{d}{d x^{4}}$ for example, convert quantities to others that no longer commute such that the result of such operations depends on the original factor ordering.

Since $c$-number considerations are often superior from the point of view of generality and elegance we will in the following use them for a first survey, and, later, we will indicate the appropriate modifications required for q-numbers. However, to avoid unnecessary repetition we will also refer to commutation relations as $c$-numbers, whereby we of course mean the corresponding Poisson brackets.

We turn now to the definition of the transformation group that the Lagrangian function permits (the precise sense to be specified shortly). We are in no way attempting in this investigation to address the most general situation. Rather, we seek a description that is sufficiently general that in the physical applications the deeper interconnections are clearly evident. We do not therefore ask for the most general group from which for a given Lagrangian identities of the type mentioned above will result. Rather we will take as our basis a special yet extended class of continuous infinite groups, which as we will show, lead to identities for an arbitrary Lagrangian (c-number-)function ${ }^{8}$.

We characterize our group through its infinitesimal transformations. We assume that both the $x^{\nu}$ and the $Q_{\alpha}$ transform in a certain way. Namely, the $\delta x^{\nu}$ (respectively the $\left.\delta Q_{\alpha}\right)$ depend on $r_{0}$ arbitrary real functions $\xi^{r}(x)\left(r=1,2, \ldots r_{0}\right)$ and their derivatives up to order $k$ (respectively $j$ ); the coefficients of these derivatives must be real, and (here is the group specialization) the $\delta x^{\nu}$ depend only on $x^{\nu}$ while the $\delta Q_{\alpha}$ depend only on $x^{\nu}$ and $Q_{\alpha}$ (and not on derivatives of the $Q_{\alpha}$ ). Explicitly [1],

$$
\left\{\begin{array}{c}
\delta x^{\nu}=a_{r}^{\nu, 0}(x) \xi^{r}(x)+a_{r}^{\nu, \sigma}(x) \frac{\partial \xi^{r}}{\partial x^{\sigma}}+a_{r}^{\nu, \sigma \ldots \tau}(x) \frac{\partial^{k} \xi^{r}}{\partial x^{\sigma} \ldots x^{\tau}}, \\
\delta Q_{\alpha}=c_{\alpha r}^{0}(x, Q) \xi^{r}(x)+c_{\alpha r}^{\sigma}(x, Q) \frac{\partial \xi^{r}}{\partial x^{\sigma}}+c_{\alpha r}^{\sigma \ldots \tau}(x, Q) \frac{\partial^{j} \xi^{r}}{\partial x^{\sigma} \ldots \partial x^{\tau}} .
\end{array}\right.
$$

In addition we have the essential assumption ${ }^{9}$,

$$
j \geq k+1 \text {. }
$$

Concerning the commutation relations relating to the functions that appear in (2), the $\xi^{r}$ must be $c$-numbers, and this property should be preserved in all transformations of the group (2) (corresponding to the understanding that the $\xi^{r}$ depend only on the $x^{\nu}$ ). Since the $a$ depend only on the $x^{\nu}$ we may also consider them to be $c$ numbers. Then the $\delta x^{\nu}$ are also $c$-numbers, as they must be in order that we may treat the $x^{\nu}$ as $c$-numbers.

The most important groups appearing in physics are of this type (cf. the second part of this work).

It remains for us to express the fact that the integral

$$
\int \mathcal{L} d x^{1} d x^{2} d x^{3} d x^{4}
$$

is invariant under the transformations (2). For this purpose we introduce a few concepts.

\footnotetext{
8 The method that is used here furthermore gives an immediate response to the general question that was just posed. For Lagrange functions that exhibit a specific form the group does not even need to be infinite in order that identities result.

${ }^{9}$ We set $\frac{\partial^{0} \xi}{(\partial x)^{0}} \equiv \xi$ and $\frac{\partial^{-1} \xi}{(\partial x)^{-1}} \equiv 0[2]$.
} 
Besides the "local" variations $\delta \Phi\left(x, Q, \frac{\partial Q}{\partial x}, \ldots\right)$ we have the "substantial" variation

$$
\delta^{*} \Phi=\delta \Phi-\frac{d \Phi}{d x^{\nu}} \delta x^{\nu}
$$

if we represent transformed quantities with a prime, then we have

$$
\delta \Phi=\Phi^{\prime}\left[x^{\prime} ; Q^{\prime}\left(x^{\prime}\right) ; \ldots\right]-\Phi[x ; Q(x) ; \ldots],
$$

while

$$
\delta^{*} \Phi=\Phi^{\prime}\left[x ; Q^{\prime}(x) ; \ldots\right]-\Phi[x ; Q(x) ; \ldots],
$$

The following important formulae result (also for q-numbers):

$$
\delta^{*} \frac{d \Phi}{d x^{\nu}}=\frac{d}{d x^{\nu}} \delta^{*} \Phi
$$

and

$$
\delta \frac{d \Phi}{d x^{\nu}}=\frac{d}{d x^{\nu}} \delta \Phi-\frac{d \Phi}{d x^{\rho}} \frac{d \delta x^{\rho}}{d x^{\nu}} .
$$

A quantity $\mathcal{K}$ is called a scalar density (with respect to the group) when it transforms with the following properties:

$$
\delta^{*} \mathcal{K}+\frac{d}{d x^{\nu}}\left(\mathcal{K} \delta x^{\nu}\right)=0
$$

or, according to (4),

$$
\delta \mathcal{K}+\mathcal{K} \frac{d \delta x^{\nu}}{d x^{\nu}}=0
$$

Quantities depend in general on two kinds of indices, first on indices $\alpha, \beta, \gamma, \ldots$ whose range is that of the index $\alpha$ in $Q_{\alpha}$. Secondly, they depend on the indices $\mu, \nu, \ldots$ which as with the index of $x^{\nu}$ range from 1 to 4 . In particular the index $r$ in $\xi^{r}$ represents one or more systems of indices of the form $(\alpha, \beta, \ldots ; \mu, \nu, \ldots)$, numbered in an arbitrary one-dimensional sequence. The indices of type $\alpha, \beta, \ldots$ could in their turn be multiple and in particular contain systems of indices $\mu, \nu, \ldots$.

A contravariant tensor $K^{\alpha \nu}$ is defined through the transformation property [3]

$$
\delta K^{\alpha \nu}=K^{\alpha \mu} \frac{d \delta x^{\nu}}{d x^{\mu}}-\underline{K^{\beta \nu} \frac{\partial \delta Q_{\beta}}{\partial Q_{\alpha}}} ;
$$

in the q-number case this definition contains an arbitrariness in the underlined term that we will remove by setting

$$
\underline{K^{\beta \nu} \frac{\partial \delta Q_{\beta}}{\partial Q_{\alpha}}}=\frac{1}{2}\left(K^{\beta \nu} \frac{\partial \delta Q_{\beta}}{\partial Q_{\alpha}}+\frac{\partial \delta Q_{\beta}}{\partial Q_{\alpha}} K^{\beta \nu \dagger}\right),
$$

where $x^{\dagger}$ is the Hermitian conjugate (adjoint) to $x$. [In the following we will use the notation

$$
\left.\underline{x}=\frac{1}{2}\left(x+x^{\dagger}\right) \cdot\right]
$$

With this assignment a Hermitian tensor remains a Hermitian tensor under an arbitrary transformation of the group.

A covariant tensor $K_{\alpha \nu}$ has the transformation property

$$
\delta K_{\alpha \nu}=-K_{\alpha \mu} \frac{d \delta x^{\mu}}{d x^{\nu}}+\underline{K_{\beta \nu} \frac{\partial \delta Q_{\alpha}}{\partial Q_{\beta}}} ;
$$

the variation of mixed tensors $K_{\alpha \beta} \ldots{ }^{\gamma \delta \cdots}{ }_{\mu \nu \ldots}{ }^{\pi \rho}$ is formed in analogy with (9) and (10). 
A tensor density $\mathcal{K}^{\alpha \nu}$ transforms as the product of a tensor $K^{\alpha \nu}$ with a scalar density $\mathcal{K}$, namely

$$
\delta \mathcal{K}^{\alpha \nu}=\mathcal{K}^{\alpha \mu} \frac{d \delta x^{\nu}}{d x^{\mu}}-\underline{\mathcal{K}^{\beta \nu} \frac{\partial \delta Q_{\beta}}{\partial Q_{\alpha}}}-\mathcal{K}^{\alpha \nu} \frac{d \delta x^{\mu}}{d x^{\mu}} .
$$

We are now in position to formulate the invariance condition with respect to the Lagrangian function. In order that the integral $\int \mathcal{L} d x^{1} d x^{2} d x^{3} d x^{4}$ be invariant, following from well-known $\operatorname{arguments}^{10}, \mathcal{L}$ must be a scalar density up to a divergence $\mathcal{L}^{\prime} \equiv \frac{d \mathcal{K}^{\nu}}{d x^{\nu}}$. Explicitly:

$$
\delta\left(\mathcal{L}+\mathcal{L}^{\prime}\right)+\left(\mathcal{L}+\mathcal{L}^{\prime}\right) \frac{d \delta x^{\nu}}{d x^{\nu}}=0
$$

Since as we have said we are not concerned with complete generality, we will be satisfied in treating in order the following characteristic cases:

1. $\mathcal{L}^{\prime}=0$, i.e., $\mathcal{L}$ is itself a scalar density:

$$
\delta \mathcal{L}+\mathcal{L} \frac{d \delta x^{\nu}}{d x^{\nu}}=0
$$

2. $\mathcal{L}^{\prime}$ contains second derivatives

$$
Q_{\alpha, \nu \rho} \equiv \frac{\partial^{2} Q_{\alpha}}{\partial x^{\nu} \partial x^{\rho}}
$$

only linearly, i.e.,

$$
\mathcal{L}^{\prime} \equiv \frac{d}{d x^{\nu}} \underline{\left[f^{\nu, \alpha \rho}(Q) Q_{\alpha, \rho}\right]}
$$

and $j=0$ (cf. Eq. (3)).

In both cases the investigation splits into two steps:

a) Implementation of the Hamiltonian method;

b) Proof of covariance of the Hamiltonian procedure under the relevant group.

We begin with the first case.

\subsection{The conjugate momenta and the identities}

Henceforth we assume condition (13) is satisfied.

First we set

$$
\mathcal{P}^{\alpha \nu}=\frac{\partial \mathcal{L}}{\partial Q_{\alpha, \nu}},
$$

and we take as momentae

$$
\mathcal{P}^{\alpha} \equiv \mathcal{P}^{\alpha 4}=\frac{\partial \mathcal{L}}{\partial \dot{Q}_{\alpha}} .
$$

We confine ourselves first to the classical $c$-number theory.

We substitute into (13) $\delta Q_{\alpha}, Q_{\alpha, \nu}$ and $\delta x^{\nu}$ through their values given in (2) and (6) as functions of $\xi^{r}$ and derivatives. That way we obtain several identities in expressing the fact that the coefficients of respective derivatives of $\xi$ have to vanish identically.

${ }_{10}$ cf. eg. E. Noether, Gött. Nach. (1918), p. 211. The divergence $\frac{d \mathcal{K}^{\nu}}{d x^{\nu}}$ appears if the integral $\int \mathcal{L} d x^{1} \ldots d x^{4}$ is not invariant for an arbitrary integration domain but only when $\mathcal{K}^{\nu}$ vanishes on the boundary. 
These identities generally contain the $\dot{Q}_{\alpha}$ not only through the just introduced functions $\mathcal{P}^{\alpha}$ but also through other relations (e.g., through the other $\mathcal{P}^{\alpha \nu}, \nu \neq 4$ ). They are not of interest in solving the system of equations (13) for $\dot{Q}_{\alpha}$; they simply represents relations that each solution $\dot{Q}_{\alpha}(Q, \mathcal{P})$ must fulfill automatically. If some of the identities under consideration contain only the $Q_{\alpha}$ (along with spatial derivatives) and the $\mathcal{P}^{\alpha}$, then the situation is fundamentally different. Then they signify that the equations (16) are not mutually independent so that the general solution will depend on arbitrary parameters (more precisely, spacetime functions).

The last case always occurs with the group (2). The highest derivatives of $\xi^{\nu}$ in $(13)$ are

$$
\frac{\partial^{j+1} \xi^{r}}{\partial x^{\sigma} \ldots \partial x^{\tau} \partial x^{\nu}}
$$

according to the assumption (3) the corresponding identities read

$$
\sum \mathcal{P}^{\alpha \nu} c_{\alpha r}^{\sigma \ldots \tau} \equiv 0^{12}
$$

where the summation runs over all permutations of the numbers $\nu, \sigma, \ldots, \tau$. For the case $\nu=\sigma=\ldots=\tau=4$ one has in particular

$$
\mathcal{P}^{\alpha} c_{\alpha r}^{44 \ldots 4} \equiv 0
$$

since the $c$ contain only the $Q_{\alpha}$ we have in (18c) $r_{0}$ identities of the last type considered that we will call "proper" identities. Furthermore it is easy to see that in general (i.e., in the case that the Lagrange function possesses no special properties ) that no more proper identities appear. Hence, the general solution $\dot{Q}_{\alpha}(Q, \mathcal{P}, \lambda)$ of $(16)$ depends on $r_{0}$ arbitrary parameters $\lambda$.

In the previous methods mentioned in the introduction one proceeded either through the destruction of the invariance properties of the Lagrangian (methods 1 and 3$)$ or through the choice of a special solution $\dot{Q}_{\alpha}\left(Q, \mathcal{P}, \lambda^{0}\right)(\operatorname{method} 2)$. In contrast the fundamental idea of the new method is to construct the Hamilton function as usual with the help of the general solution $\dot{Q}_{\alpha}(Q, \mathcal{P}, \lambda)$ with undetermined $\lambda^{r}$, without for the moment worrying about the proper identities. Field equations and commutation relations have the canonical form, with the former containing the $\lambda^{r}$. In this canonical formalism the proper identities ultimately become constraints. We will see that in addition to its simplicity the method has the big advantage that the proof of covariance can be carried out without difficulty.

\subsection{Transition to q-numbers}

We must first investigate how the relations described above have to be refashioned in passing to q-numbers. According to (1) the relation (15) reads

$$
\mathcal{P}^{\alpha \nu}=\frac{1}{2}\left(p^{\alpha \nu}+p^{\alpha \nu \dagger}\right)=\underline{p^{\alpha \nu}}
$$

with

$$
p^{\alpha \nu}=\mathcal{A}^{\alpha \nu ; \beta \mu} Q_{\beta, \mu}+\mathcal{B}^{\alpha \nu}
$$

\footnotetext{
${ }^{11}$ Equation numbers appear with an appended $c$ when they have unlimited validity only for $c$-numbers.
} 
A bar over an index of the form $\mu: \bar{\mu}$ signifies that the index ranges from 1 to 3 ; the summation convention will also hold for barred indices. With this notation according to (19) and (20) we write

$$
\left\{\begin{array}{c}
\mathcal{P}^{\alpha}=\underline{p}^{\alpha} \\
p^{\alpha}=\mathcal{A}^{\alpha \beta} \dot{Q}_{\beta}+\mathcal{D}^{\alpha},
\end{array}\right.
$$

where we define

$$
\left\{\begin{array}{c}
\mathcal{A}^{\alpha \beta} \equiv \mathcal{A}^{\alpha 4 ; \beta 4}, \\
\mathcal{D}^{\alpha} \equiv \mathcal{A}^{\alpha 4 ; \beta \bar{\mu}} Q_{\beta, \bar{\mu}}+\mathcal{B}^{\alpha 4} .
\end{array}\right.
$$

We assume in this equation that

$$
\mathcal{A}^{\alpha \nu ; \beta \mu}=\mathcal{A}^{\beta \mu ; \alpha \nu}
$$

and in particular that

$$
\mathcal{A}^{\alpha \beta}=\mathcal{A}^{\beta \alpha}
$$

which implies of course no restriction.

The considerations of the previous paragraph yield instead of (17c) and (18c)

$$
\sum \underline{c_{\alpha r}^{\sigma \ldots \tau} p^{\alpha \nu}} \equiv 0
$$

and

$$
\underline{c_{\alpha r}^{4 \ldots 4} p^{\alpha}} \equiv 0
$$

Since in particular (24) holds identically in the $\dot{Q}_{\alpha}$ we have according to (21)

$$
\begin{aligned}
& c_{\alpha r}^{4 \ldots 4} \mathcal{A}^{\alpha \beta}=0, \\
& c_{\alpha r}^{4 \ldots 4} \mathcal{D}^{\alpha}=0 ;
\end{aligned}
$$

the coefficients in the Lagrange function must satisfy, among others, these conditions in order that it possess the required density property. We emphasize the relations (25) and (26) for later use.

We cannot proceed further without knowing something about the commutation relations $\left[Q_{\alpha}, \dot{Q}_{\beta}\right]$. If we were to know the $\dot{Q}_{\beta}$ as functions of the $Q_{\alpha}$ and $\mathcal{P}^{\alpha}$, then we would be able to derive the value of $\left[Q_{\alpha}, \dot{Q}_{\beta}\right]$ from the canonical commutation relations, which, as we have said, we wish to retain. But it is not even clear a priori whether we can from (21) derive the $\dot{Q}_{\beta}$ as functions of the matrices $\mathcal{P}^{\alpha}$, or only as functions of the matrix elements of $\mathcal{P}^{\alpha}$. The only way we can overcome this problem is to make tentatively an assumption about the $\left[Q_{\alpha}, \dot{Q}_{\beta}\right]$ on grounds of which the solution of $(21)$ takes the form $\dot{Q}_{\alpha}(Q, \mathcal{P}, \lambda)$ and to check later whether the assumption is compatible with canonical commutation relations.

A selfevident assumption is the following: the $\left[Q_{\alpha}, \dot{Q}_{\beta}\right]$ should be anti-Hermitian ${ }^{12}$ functions of $Q_{\alpha}$ and $Q_{\alpha, \bar{\nu}}$, but not of the $\dot{Q}_{\alpha}$ (respectively the $\mathcal{P}^{\alpha}$ ) [4] (Whether undetermined factors like $\delta(0)$ appear when $Q_{\alpha}$ and $\dot{Q}_{\beta}$ are taken at the same location is irrelevant). We now quote a few immediate conclusions from these assumptions:

1. According to $(20)$ both $\left[Q_{\alpha}, p^{\beta \nu}\right]$ and $\left[Q_{\alpha}, p^{\beta \nu \dagger}\right]$ are anti-Hermitian functions of the $Q_{\alpha}$ and the $Q_{\alpha, \bar{\nu}}$ alone.

2. The $\left[Q_{\alpha}, \dot{Q}_{\beta}\right],\left[Q_{\alpha}, p^{\beta \nu}\right]$ and $\left[Q_{\alpha}, p^{\beta \nu \dagger}\right]$ commute with every function of the $Q_{\alpha}$ and $Q_{\alpha, \bar{\nu}}$.

$\overline{12}$ A q-number $x$ is said to be anti-Hermitian when $x^{\dagger}=-x$. 
3. We have

$$
\left[Q_{\alpha}, p^{\beta \nu}\right]=\left[Q_{\alpha}, p^{\beta \nu \dagger}\right]
$$

Therefore instead of (23) and (24) we can write

$$
\begin{gathered}
\sum \underline{c_{\alpha r}^{\sigma \ldots \tau} \mathcal{P}^{\alpha \nu}}=0, \\
\mathcal{F}_{r} \equiv \underline{c_{\alpha r}^{4 \ldots 4} \mathcal{P}^{\alpha}}=0 .
\end{gathered}
$$

From (25) it follows that the $N$ linear equations

$$
\mathcal{A}^{\alpha \beta} \dot{Q}_{\beta}+\dot{Q}_{\beta} \mathcal{A}^{\beta \alpha}=2\left(\mathcal{P}^{\alpha}-\mathcal{D}^{\alpha}\right),
$$

are not independent, rather that the determinant $\left|\mathcal{A}^{\alpha \beta}\right|$ has the rank $N-r_{0}$. Since it is symmetric there exists a nonvanishing principal minor of degree $N-r_{0}$; we will denote the corresponding indices with a prime:

$$
\left|\mathcal{A}^{\alpha^{\prime} \beta^{\prime}}\right| \neq 0
$$

while the remaining indices will be doubly primed: $\mu^{\prime \prime}, \nu^{\prime \prime}, \ldots$ The determinant $\left|\mathcal{A}^{\alpha^{\prime} \beta^{\prime}}\right|$ as well as its reciprical $\left|\mathcal{A}_{\alpha^{\prime} \beta^{\prime}}\right|$ are symmetric, and we have

$$
\mathcal{A}^{\alpha^{\prime} \beta^{\prime}} \mathcal{A}_{\beta^{\prime} \gamma^{\prime}}=\delta_{\gamma^{\prime}}^{\alpha^{\prime}}
$$

where as usual $\delta_{\gamma}^{\alpha}$ equals 0 or 1 depending on $\alpha \neq \beta$ or $\alpha=\beta$.

If we succeed in finding a special solution $\dot{Q}_{\beta}^{0}(Q, \mathcal{P})$ of $(21)$, then the most general solution has the form:

$$
\dot{Q}_{\beta}=\dot{Q}_{\beta}^{0}+\lambda^{r} x_{\beta r},
$$

where the $\lambda^{r}$ are $r_{0}$ arbitrary parameters and the $x_{\beta r}$ represent $r_{0}$ independent solutions of the homogeneous equations

$$
\mathcal{A}^{\alpha \beta} x_{\beta r}+x_{\beta r} \mathcal{A}^{\beta \alpha}=0 .
$$

According to (25) we can now choose

$$
x_{\beta r}=c_{\beta r}^{4 \ldots 4}
$$

and write

$$
\dot{Q}_{\beta}=\dot{Q}_{\beta}^{0}+\lambda^{r} c_{\beta r}^{4 \ldots 4}
$$

Furthermore I maintain that

$$
\left\{\begin{array}{l}
\dot{Q}_{\beta^{\prime}}^{0}=\frac{1}{2}\left\{\mathcal{A}_{\beta^{\prime} \gamma^{\prime}}\left(\mathcal{P}^{\gamma^{\prime}}-\mathcal{D}^{\gamma^{\prime}}\right)+\left(\mathcal{P}^{\gamma^{\prime}}-\mathcal{D}^{\gamma^{\prime}}\right) \mathcal{A}_{\gamma^{\prime} \beta^{\prime}}\right\} \\
\dot{Q}_{\beta^{\prime \prime}}^{0}=0
\end{array}\right.
$$

is a special solution of (21). If this is verified, then we have actually succeeded in solving (21) for the $\dot{Q}_{\beta}$ : the solution (31) manifestly has the required property since by virtue of the canonical commutation relations $\left[Q_{\alpha}, \dot{Q}_{\beta}\right]$ is an anti-Hermitian function of the $Q_{\alpha}$ and $Q_{\alpha, \bar{\mu}}$. 
Substituting (32) into the left hand side of (21) [5], which for the moment we will call $\mathcal{T}_{\alpha}$, one obtains

$$
\begin{aligned}
\mathcal{T}^{\alpha}= & \frac{1}{2} \mathcal{A}^{\alpha \beta^{\prime}}\left\{\mathcal{A}_{\beta^{\prime} \gamma^{\prime}}\left(\mathcal{P}^{\gamma^{\prime}}-\mathcal{D}^{\gamma^{\prime}}\right)+\left(\mathcal{P}^{\gamma^{\prime}}-\mathcal{D}^{\gamma^{\prime}}\right) \mathcal{A}_{\gamma^{\prime} \beta^{\prime}}\right\} \\
& +\frac{1}{2}\left\{\mathcal{A}_{\beta^{\prime} \gamma^{\prime}}\left(\mathcal{P}^{\gamma^{\prime}}-\mathcal{D}^{\gamma^{\prime}}\right)+\left(\mathcal{P}^{\gamma^{\prime}}-\mathcal{D}^{\gamma^{\prime}}\right) \mathcal{A}_{\gamma^{\prime} \beta^{\prime}}\right\} \mathcal{A}^{\beta^{\prime} \alpha} \\
= & \mathcal{A}^{\alpha \beta^{\prime}} \mathcal{A}_{\beta^{\prime} \gamma^{\prime}}\left(\mathcal{P}^{\gamma^{\prime}}-\mathcal{D}^{\gamma^{\prime}}\right)+\left(\mathcal{P}^{\gamma^{\prime}}-\mathcal{D}^{\gamma^{\prime}}\right) \mathcal{A}_{\gamma^{\prime} \beta^{\prime}} \mathcal{A}^{\beta^{\prime} \alpha} \\
& +\frac{1}{2} \mathcal{A}^{\alpha \beta^{\prime}}\left[\mathcal{P}^{\gamma^{\prime}}-\mathcal{D}^{\gamma^{\prime}}, \mathcal{A}_{\gamma^{\prime} \beta^{\prime}}\right]+\frac{1}{2}\left[\mathcal{A}_{\beta^{\prime} \gamma^{\prime}}, \mathcal{P}^{\gamma^{\prime}}-\mathcal{D}^{\gamma^{\prime}}\right] \mathcal{A}^{\beta^{\prime} \alpha} \\
= & \mathcal{A}^{\alpha \beta^{\prime}} \mathcal{A}_{\beta^{\prime} \gamma^{\prime}}\left(\mathcal{P}^{\gamma^{\prime}}-\mathcal{D}^{\gamma^{\prime}}\right)+\left(\mathcal{P}^{\gamma^{\prime}}-\mathcal{D}^{\gamma^{\prime}}\right) \mathcal{A}_{\gamma^{\prime} \beta^{\prime}} \mathcal{A}^{\beta^{\prime} \alpha}
\end{aligned}
$$

by virtue of the second implication of our assumption [6]. For $\alpha=\alpha^{\prime}$ we deduce already from (30)

$$
\mathcal{T}^{\alpha^{\prime}}=2\left(\mathcal{P}^{\alpha^{\prime}}-\mathcal{D}^{\alpha^{\prime}}\right)
$$

Now according to the theory of linear equations and using our assumption regarding $\left[Q_{\alpha}, \dot{Q}_{\beta}\right]$ the identities $(29)$ are equivalent to

$$
\mathcal{P}^{\alpha^{\prime \prime}}=\underline{\mathcal{A}^{\alpha^{\prime \prime} \beta^{\prime}} \mathcal{A}_{\beta^{\prime} \gamma^{\prime}} \mathcal{P}^{\gamma^{\prime}}}
$$

and in the same manner $(26)$ is equivalent to

$$
\mathcal{D}^{\alpha^{\prime \prime}}=\mathcal{A}^{\alpha^{\prime \prime} \beta^{\prime}} \mathcal{A}_{\beta^{\prime} \gamma^{\prime}} \mathcal{D}^{\gamma^{\prime}}
$$

It follows that also

$$
\mathcal{T}^{\alpha^{\prime \prime}}=2\left(\mathcal{P}^{\alpha^{\prime \prime}}-\mathcal{D}^{\alpha^{\prime \prime}}\right)
$$

whereby the proof is completed that (31), (32) represent the most general solution of (21) in agreement with the canonical commutation relations.

\subsection{Construction of the Hamiltonian}

Classically the Hamiltonian reads

$$
\mathcal{H}=\mathcal{P}^{\alpha} \dot{Q}_{\alpha}-\mathcal{L}
$$

in every quantum mechanical approach we must demand that

$$
\frac{\partial \mathcal{H}}{\partial Q_{\alpha, \bar{\nu}}}=-\frac{\partial \mathcal{L}}{\partial Q_{\alpha, \bar{\nu}}},
$$

a property that will prove itself indispensible in the elaboration of the theory [7].

We have

$$
\left(\frac{\partial \mathcal{L}}{\partial Q_{\alpha, \bar{\nu}}}\right)_{\mathcal{P}^{\alpha}}=\left(\frac{\partial \mathcal{L}}{\partial Q_{\alpha, \bar{\nu}}}\right)_{\dot{Q}_{\alpha}}+\underline{\left(\frac{\partial \dot{Q}_{\beta}}{\partial Q_{\alpha, \bar{\nu}}}\right)_{\mathcal{P}^{\alpha}} p^{\beta}}
$$

and since according to $\left(31\right.$ and $(32),\left(\frac{\partial \dot{Q}_{\beta}}{\partial Q_{\alpha, \bar{\nu}}}\right)_{\mathcal{P}^{\alpha}}$ does not contain the $\mathcal{P}^{\alpha}$ we can write

$$
\left(\frac{\partial \mathcal{L}}{\partial Q_{\alpha, \bar{\nu}}}\right)_{\mathcal{P}^{\alpha}}=\left(\frac{\partial \mathcal{L}}{\partial Q_{\alpha, \bar{\nu}}}\right)_{\dot{Q}_{\alpha}}+\left(\frac{\partial \dot{Q}_{\beta}}{\partial Q_{\alpha, \bar{\nu}}}\right)_{\mathcal{P}^{\alpha}} \mathcal{P}^{\beta}
$$


The ansatz

$$
\mathcal{H}=\underline{\dot{Q}_{\alpha} \mathcal{P}^{\alpha}}-\mathcal{L}
$$

therefore satisfies the desired condition (33). Since by (25) and (26)

$$
\mathcal{L}[Q ; \dot{Q}(Q, \mathcal{P}, \lambda)]=\mathcal{L}\left(Q, \dot{Q}^{0}\right),
$$

using the notation (29) we can write

$$
\mathcal{H}=\mathcal{H}_{0}+\lambda^{r} \mathcal{F}_{r}
$$

with

$$
\mathcal{H}_{0}=\underline{\dot{Q}_{\alpha}^{0} \mathcal{P}^{\alpha}}-\mathcal{L}\left[Q, \dot{Q}^{0}(Q, \mathcal{P})\right]
$$

Now we set the canonical commutation relations

$$
\left\{\begin{array}{c}
{\left[Q_{\alpha}(\mathbf{r}), Q_{\beta}\left(\mathbf{r}^{\prime}\right)\right]=\left[\mathcal{P}^{\alpha}(\mathbf{r}), \mathcal{P}^{\beta}\left(\mathbf{r}^{\prime}\right)\right]=0} \\
{\left[\mathcal{P}^{\alpha}(\mathbf{r}), Q_{\beta}\left(\mathbf{r}^{\prime}\right)\right]=\omega \delta_{\beta}^{\alpha} \delta\left(\mathbf{r}-\mathbf{r}^{\prime}\right), \omega=\frac{h c}{2 \pi i},}
\end{array}\right.
$$

as well as the field equations

$$
\begin{aligned}
& {\left[\overline{\mathcal{H}}, Q_{\alpha}\right]=\omega \dot{Q}_{\alpha},} \\
& {\left[\overline{\mathcal{H}}, \mathcal{P}^{\alpha}\right]=\omega \dot{\mathcal{P}}^{\alpha},}
\end{aligned}
$$

where we use the notation

$$
\overline{\mathcal{A}} \equiv \int \mathcal{A} d x^{1} d x^{2} d x^{3}
$$

the integration domain must be chosen in such a manner that field quantities assume a constant value on the boundary, indeed, such values that $\mathcal{L}$ vanishes there [8].

In addition to (37) and (38) we have the proper identities (29) $\mathcal{F}_{r}=0$ as constraints. But it must be proven that it is permissible to set all of the $\mathcal{F}_{r}$ simultaneously to zero; in other words, that the $\mathcal{F}_{r}$ commute with each other, at least on account of the constraints $\mathcal{F}_{r}=0$ themselves.

The following observations will serve not only this purpose, but are also the basis for the proof of covariance to be adduced later on.

We define first the energy-momentum pseudo tensor ${ }^{13}$

$$
\mathcal{G}_{\mu}^{\nu}=\underline{\mathcal{P}^{\alpha \nu} Q_{\alpha, \mu}}-\delta_{\mu}^{\nu} \mathcal{L}
$$

and then the energy-momentum pseudo density is

$$
\mathcal{G}_{\mu}=\mathcal{G}_{\mu}^{4}=\underline{\mathcal{P}^{\alpha} Q_{\alpha, \mu}}-\delta_{\mu}^{4} \mathcal{L}
$$

whose fourth pseudo component is the Hamiltonian function (34):

$$
\mathcal{H}=\mathcal{G}_{4}=\mathcal{G}_{4}^{4} .
$$

The components of the total momentum are then $\overline{\mathcal{G}_{\bar{\nu}}}$ and the total energy is $\overline{\mathcal{H}}$.

The CR (commutation relations) of $\overline{\mathcal{H}}$ with the $Q_{\alpha}, \mathcal{P}^{\alpha}$ are given by (38). Concerning the $\overline{\mathcal{G}}_{\bar{\mu}}$ we first find referring to (37) that

$$
\left\{\begin{array}{l}
{\left[\mathcal{G}_{\bar{\nu}}(\mathbf{r}), Q_{\alpha}\left(\mathbf{r}^{\prime}\right)\right]=\omega \frac{\partial Q_{\alpha}}{\partial x^{\bar{\nu}}} \delta\left(\mathbf{r}-\mathbf{r}^{\prime}\right),} \\
{\left[\mathcal{G}_{\bar{\nu}}(\mathbf{r}), \mathcal{P}^{\alpha}\left(\mathbf{r}^{\prime}\right)\right]=-\omega \mathcal{P}^{\alpha} \frac{\partial \delta\left(\mathbf{r}-\mathbf{r}^{\prime}\right)}{\partial x^{\bar{\nu}}}}
\end{array}\right.
$$

\footnotetext{
13 The prefix "pseudo" signifies that the relevant quantitie are not tensors.
} 
then

$$
\left[\overline{\mathcal{G}}_{\bar{\nu}}, \Phi(Q, \mathcal{P})\right]=\omega \frac{d \Phi}{d x^{\bar{\nu}}}
$$

and therefore more generally,

$$
\omega \frac{d \Phi}{d x^{\bar{\nu}}}=\left[\overline{\mathcal{G}}_{\bar{\mu}}, \Phi(Q, \mathcal{P}, x)\right]+\omega \frac{\partial \Phi}{\partial x^{\bar{\nu}}} .
$$

From this it follows immediately that [9]

$$
\left[\overline{\mathcal{G}}_{\mu}, \overline{\mathcal{G}}_{\nu}\right]=0:
$$

this constitutes an expression for the commutability of the derivatives $\frac{d}{d x^{\nu}}$ whose physical content is the constancy in time of the $\overline{\mathcal{G}_{\mu}}$ that follows from equations (38), $(37)^{14}$.

\subsection{Quantum-mechanical expression of the infinitesimal transformations of the group}

In this paragraph we prove the the proposition:

$$
\omega \delta^{*} \Phi(Q, \mathcal{P})=[\overline{\mathcal{M}}, \Phi]
$$

where

$$
\mathcal{M}=\underline{\mathcal{P}^{\alpha} \delta Q_{\alpha}}-\mathcal{G}_{\mu} \delta x^{\mu}
$$

This should hold on account of the field equations (38) and the CR (31), under the assumption that $\mathcal{L}$ is a scalar density.

To prove this proposition it will suffice to show that

$$
\left\{\begin{array}{l}
\omega \delta^{*} Q_{\alpha}=\left[\overline{\mathcal{M}}, Q_{\alpha}\right], \\
\omega \delta^{*} \mathcal{P}^{\alpha}=\left[\overline{\mathcal{M}}, \mathcal{P}^{\alpha}\right] .
\end{array}\right.
$$

According to (37) and (42), considering that by (2) $\delta Q_{\alpha}$ contains only the $Q_{\alpha}$ (and not the $\left.\mathcal{P}^{\alpha}\right)$,

$$
\left[\overline{\mathcal{M}}, Q_{\alpha}\right]=\omega \delta Q_{\alpha}-\frac{d Q_{\alpha}}{d x^{\bar{\mu}}} \delta x^{\bar{\mu}}-\left[\overline{\delta x^{4} \mathcal{H}}, Q_{\alpha}\right]
$$

Now according to H.P.I, equation (20),

$$
\left\{\begin{aligned}
{\left[\overline{\delta x^{4} \mathcal{H}}, Q_{\alpha}\right] } & =\omega \frac{\partial\left(\delta x^{4} \mathcal{H}\right)}{\partial \mathcal{P}^{\alpha}}=\delta x^{4}\left[\overline{\mathcal{H}}, Q_{\alpha}\right], \\
{\left[\overline{\delta x^{4} \mathcal{H}}, \mathcal{P}^{\alpha}\right] } & =-\omega\left\{\frac{\partial\left(\delta x^{4} \mathcal{H}\right)}{\partial Q_{\alpha}}-\frac{d}{d x^{\bar{\nu}}} \frac{\partial\left(\delta x^{4} \mathcal{H}\right)}{\partial Q_{\alpha, \bar{\nu}}}\right\} \\
& =\delta x^{4}\left[\overline{\mathcal{H}}, \mathcal{P}^{\alpha}\right]+\omega \frac{d \delta x^{4}}{d x^{\bar{\nu}}} \frac{\partial \mathcal{H}}{\partial Q_{\alpha, \bar{\nu}}} .
\end{aligned}\right.
$$

The first relation (47) therefore follows referring to (4), using in addition the first field equation (38).

Similarly, referring to the second field equation (38) and equation (33) one finds

$$
\left\{\begin{aligned}
\frac{1}{\omega}\left[\overline{\mathcal{M}}, \mathcal{P}^{\alpha}\right]= & -\frac{\mathcal{P}^{\beta} \frac{\partial \delta Q_{\beta}}{\partial Q_{\alpha}}-\frac{d}{d x^{\bar{\nu}}}\left(\mathcal{P}^{\alpha} \delta x^{\bar{\nu}}\right)}{} \\
& -\frac{d \mathcal{P}^{\alpha}}{d x^{4}} \delta x^{4}+\mathcal{P}^{\alpha \bar{\nu}} \frac{d \delta x^{4}}{d x^{\bar{\nu}}} \\
= & -\mathcal{P}^{\beta} \frac{\partial \delta Q_{\beta}}{\partial Q_{\alpha}}+\mathcal{P}^{\alpha \nu} \frac{d \delta x^{4}}{d x^{\nu}}-\frac{d}{d x^{\nu}}\left(\mathcal{P}^{\alpha} \delta x^{\nu}\right) .
\end{aligned}\right.
$$

$\overline{14}$ In case the $\lambda^{r}$ contain the $x^{4}$ explicitly, (44) holds only because of the constraints (29). 
It remains only to show that the right hand side of (49) is equal to $\delta^{*} \mathcal{P}^{\alpha}$. We therefore calculate $\delta \mathcal{P}^{\alpha}$ directly, or more generally $\delta \mathcal{P}^{\alpha \nu}$. First we have

$$
\delta \mathcal{P}^{\alpha \nu}=\delta\left(\frac{\partial \mathcal{L}}{\partial Q_{\alpha, \nu}}\right)=\frac{\partial(\delta \mathcal{L})}{\partial Q_{\alpha, \nu}}-\frac{\partial \mathcal{L}}{\partial Q_{\beta, \mu}} \frac{\partial \delta Q_{\beta, \mu}}{\partial Q_{\alpha, \nu}},
$$

for $c$-numbers in general, and certainly for q-numbers whenever $\mathcal{L}$ has the form $(1)$ and $\frac{\partial \delta Q_{\beta, \mu}}{\partial Q_{\alpha, \nu}}$ does not contain the $\dot{Q}_{\alpha}$ (respectively, the $\left.\mathcal{P}^{\alpha}\right)$. The latter is true in our case according to the formula (6) that gives

$$
\begin{aligned}
\frac{\partial}{\partial Q_{\alpha, \nu}} \delta Q_{\beta, \mu} & =\frac{\partial}{\partial Q_{\alpha, \nu}}\left\{\frac{d}{d x^{\mu}} \delta Q_{\beta}-Q_{\beta, \rho} \frac{d \delta x^{\rho}}{d x^{\mu}}\right\} \\
& =\frac{\partial \delta Q_{\beta}}{\partial Q_{\alpha}} \delta_{\mu}^{\nu}-\frac{d \delta x^{\nu}}{d x^{\mu}} \delta_{\beta}^{\alpha} .
\end{aligned}
$$

Substituting this into (50) yields

$$
\delta \mathcal{P}^{\alpha \nu}=\underline{-\mathcal{P}^{\beta \nu} \frac{\partial \delta Q_{\beta}}{\partial Q_{\alpha}}}+\mathcal{P}^{\alpha \mu} \frac{d \delta x^{\nu}}{d x^{\mu}}+\frac{\partial(\delta \mathcal{L})}{\partial Q_{\alpha, \nu}} ;
$$

now using (13) we deduce

$$
\delta \mathcal{P}^{\alpha \nu}=\underline{-\mathcal{P}^{\beta \nu} \frac{\partial \delta Q_{\beta}}{\partial Q_{\alpha}}}+\mathcal{P}^{\alpha \mu} \frac{d \delta x^{\nu}}{d x^{\mu}}-\mathcal{P}^{\alpha \nu} \frac{d \delta x^{\mu}}{d x^{\mu}} ;
$$

i.e., as the comparison with (11) instructs us: $\mathcal{P}^{\alpha \nu}$ is a tensor density. From (51) with reference to (4) for $\delta^{*} \mathcal{P}^{\alpha} \equiv \delta^{*} \mathcal{P}^{\alpha 4}$ the expression (49) follows immediately. The formula (45) is hereby proven.

\subsection{The $\overline{\mathcal{F}_{r}}$ as special infinitesimal transformations}

We consider a fixed but arbitrary slice $x^{4}=x_{0}^{4}$. On this slice we consider the transformations of our group (2) that are defined through the conditions [10]

$$
\left\{\begin{array}{c}
\left(\xi^{r}\right)_{x^{4}=x_{0}^{4}}=\left(\frac{\partial \xi^{r}}{\partial x^{\sigma}}\right)_{x^{4}=x_{0}^{4}}=\ldots=\left(\frac{\partial^{j-1} \xi^{r}}{\partial x^{\sigma} \ldots \partial x^{\tau}}\right)_{x^{4}=x_{0}^{4}}=0 \\
\left(\frac{\partial^{j} \xi^{r}}{\partial x^{\sigma} \ldots \partial x^{\tau}}\right)_{x^{4}=x_{0}^{4}}=0, \text { if all of the } \sigma \ldots \tau \text { are not equal to } 4 \\
\left(\frac{\partial^{j} \xi^{r}}{\left(\partial x^{4}\right)^{j}}\right)_{x^{4}=x_{0}^{4}}=\epsilon^{r}
\end{array}\right.
$$

where the $\epsilon^{r}$ are arbitrary spatial functions.

On account of the assumption (3) these transformations do not lead out of the $x^{4}=x_{0}^{4}$ slice. They constitute at every point in this slice a finite continuous subgroup of the group (2), whose infinitesimal transformations are given by

$$
\omega \delta^{\prime} \Phi(Q, \mathcal{P})=\left[\overline{\epsilon^{r} \mathcal{F}_{r}}, \Phi\right]
$$

(The $Q, \mathcal{P}, \mathcal{F}$ are hereby taken at $x^{4}=x_{0}^{4}$.)

Lie's second theorem on finite transformation groups declares when applied to this subgroup that at every point of the slice

$$
\left[\mathcal{F}_{r},\left[\mathcal{F}_{s}, \Phi\right]\right]-\left[\mathcal{F}_{s},\left[\mathcal{F}_{r}, \Phi\right]\right]=c_{r s}^{t}\left[\mathcal{F}_{t}, \Phi\right]
$$


where the $c_{r s}^{t}$ are the point $\left(x^{1}, x^{2}, x^{3}, x_{0}^{4}\right)$ dependent group "structure constants". By the Jacobi bracket identity the left hand side is simply equal to

$$
\left[\left[\mathcal{F}_{s}, \mathcal{F}_{r}\right], \Phi\right]
$$

we therefore obtain [11]

$$
\left[\mathcal{F}_{s}, \mathcal{F}_{r}\right]=c_{r s}^{t} \mathcal{F}_{t}
$$

From here follows the still needed fact required for the establishment of the method presented in section 4 that because of $\mathcal{F}_{r}=0$ the $\mathcal{F}_{r}$ must commute with each other.

\subsection{The infinitesimal transformation $\overline{\mathcal{M}}$ as integral of the motion}

Let us return for the moment to the pure $c$-number theory. We set

$$
\mathcal{M}^{\nu}=\underline{\mathcal{P}^{\alpha \nu} \delta Q_{\alpha}}-\mathcal{G}_{\mu}^{\nu} \delta x^{\mu}
$$

and

$$
\mathcal{L}^{\alpha}=\frac{\partial \mathcal{L}}{\partial Q_{\alpha}}-\frac{d}{d x^{\nu}} \frac{\partial \mathcal{L}}{\partial Q_{\alpha, \nu}},
$$

so is easy to see, the assumption (13) is equivalent to

$$
\frac{d \mathcal{M}^{\nu}}{d x^{\nu}}+\mathcal{L}^{\alpha} \delta^{*} Q_{\alpha}=0
$$

taking into account that according to (46) and (54)

$$
\mathcal{M} \equiv \mathcal{M}^{4}
$$

and using the notation (39) we have [12]

$$
\frac{\overline{d \mathcal{M}^{\bar{\nu}}}}{d x^{\bar{\nu}}}=0
$$

then it follows from $(56 \mathrm{c})$ that

$$
\frac{d \overline{\mathcal{M}}}{d x^{4}}=-\overline{\mathcal{L}^{\alpha} \delta^{*} Q_{\alpha}}
$$

Now it is well-known that the Hamiltonian equations (38) (by virtue of the proper identities (29)] are equivalent to the Lagrangian equations

$$
\mathcal{L}^{\alpha}=0
$$

Consequently by (57c), due to (13) and (38),

$$
\frac{d \overline{\mathcal{M}}}{d x^{4}}=0
$$

holds.

Equation (56c) cannot be carried over to q-numbers. Nevertheless the derivation of (58) succeeds through use of the very same assumptions (13) and (38), only under somewhat different circumstances. Both relations (13) and (38) were essential 
in the derivation of (43) and (45). Let us apply these latter results to the identity (5), whereby we let $\Phi$ depend only on $Q$ and $\mathcal{P}$ :

$$
\begin{gathered}
{\left[\overline{\mathcal{M}},\left[\overline{\mathcal{G}}_{\nu}, \Phi\right]\right]=\left[\overline{\mathcal{G}}_{\nu},[\overline{\mathcal{M}}, \Phi]\right]+\left[\omega \frac{\partial \overline{\mathcal{M}}}{\partial x^{\nu}}, \Phi\right]} \\
{\left[\left[\overline{\mathcal{G}}_{\nu}, \overline{\mathcal{M}}\right]+\omega \frac{\partial \overline{\mathcal{M}}}{\partial x^{\nu}}, \Phi\right]=0}
\end{gathered}
$$

using the Jacobian identity, or finally

$$
\left[\frac{d \overline{\mathcal{M}}}{d x^{\nu}}, \Phi\right]=0
$$

In particular $\frac{d \overline{\mathcal{M}}}{d x^{4}}$ is a $c$-number (generally dependent on $x^{4}$ ). But this $c$-number, as a sum of q-numbers only, can be nothing other than zero. This conclusion is confirmed through a somewhat wearysome calculation of $\frac{d \overline{\mathcal{M}}}{d x^{4}}$.

Interesting conclusions regarding the relation between $\overline{\mathcal{M}}$ and the functions $\mathcal{F}_{r}$ can be drawn from (58). Under integration by parts $\overline{\mathcal{M}}$ takes the form

$$
\overline{\mathcal{M}}=\int d x^{1} d x^{2} d x^{3} \sum_{i=0}^{i=j} \mathcal{N}_{r}^{i} \frac{\partial^{i} \xi^{r}}{\left(\partial x^{4}\right)^{i}}
$$

where

$$
\mathcal{N}_{r}^{j} \equiv \mathcal{F}_{r}
$$

Equation (58) is then put as follows:

$$
\int d x^{1} d x^{2} d x^{3} \sum_{i=0}^{i=j} \mathcal{N}_{r}^{i} \frac{\partial^{i+1} \xi^{r}}{\left(\partial x^{4}\right)^{i+1}}=-\int d x^{1} d x^{2} d x^{3} \sum_{i=0}^{i=j} \frac{d \mathcal{N}_{r}^{i}}{d x^{4}} \frac{\partial^{i} \xi^{r}}{\left(\partial x^{4}\right)^{i}}
$$

From there one concludes through comparison of coefficients that

$$
\mathcal{N}_{r}^{i}=-\frac{d \mathcal{N}_{r}^{i+1}}{d x^{4}}(i=0,1, \ldots, j-1),
$$

and

$$
\mathcal{N}_{r}^{j}=0, \frac{d \mathcal{N}_{r}^{0}}{d x^{4}}=0
$$

(60) and (61) yield

$$
\mathcal{N}_{r}^{i}=(-1)^{j-i} \frac{d^{j-i} \mathcal{F}_{r}}{\left(d x^{4}\right)^{j-i}}(i=0,1, \ldots, j),
$$

and $\overline{\mathcal{M}}$ therefore assumes the remarkable form

$$
\overline{\mathcal{M}}=\int d x^{1} d x^{2} d x^{3} \sum_{i=0}^{i=j}(-1)^{j-i} \frac{d^{j-i} \mathcal{F}_{r}}{\left(d x^{4}\right)^{j-i}} \frac{\partial^{i} \xi^{r}}{\left(\partial x^{4}\right)^{i}} .
$$

The first identity (62) is according (60) trivially $\mathcal{F}_{r}=0$, the second shows, however, that on account of the field equations and the identities (29)

$$
\frac{d^{j+1} \mathcal{F}_{r}}{\left(d x^{4}\right)^{j+1}}=0
$$


This provides the answer to the question to what extent differentiation of the constraints (29) yields new relations [13].

If in particular $j=1$, then the only new equations are $\frac{d \mathcal{F}_{r}}{d x^{4}}=0$, i.e.,

$$
\left[\overline{\mathcal{H}}, \mathcal{F}_{r}\right]+\omega \frac{\partial \mathcal{F}_{r}}{\partial x^{4}}=0
$$

If the Lagrangian is of the form (1), i.e., if (35) holds, then referring to (53) this last equation becomes

$$
\left[\overline{\mathcal{H}_{0}}, \mathcal{F}_{r}\right]+\omega \frac{\partial \mathcal{F}_{r}}{\partial x^{4}}+c_{r s}^{t} \lambda^{s} \mathcal{F}_{t}=0
$$

or since $\mathcal{F}_{r}=0$

$$
\left[\overline{\mathcal{H}_{0}}, \mathcal{F}_{r}\right]+\omega \frac{\partial \mathcal{F}_{r}}{\partial x^{4}}=0
$$

since neither the constraints or the new equations contain the $\lambda^{r}$ the latter remain essentially undetermined. (This is however different in case $j>1$ since then already the $\frac{d^{2} \mathcal{F}_{r}}{\left(d x^{4}\right)^{2}}$ contain the $\lambda^{r}$.) As a consequence of the essential indeterminateness of the $\lambda^{r}, r_{0}$ field equations of the form

$$
\omega \dot{\mathcal{P}}^{\alpha}=\left[\overline{\mathcal{H}}, \mathcal{P}^{\alpha}\right]
$$

are missing [14]. The equations

$$
\mathcal{N}_{r}^{0} \equiv \frac{d \mathcal{F}_{r}}{d x^{4}}=0, \text { i.e. }\left[\overline{\mathcal{H}_{0}}, \mathcal{F}_{r}\right]+\omega \frac{\partial \mathcal{F}_{r}}{\partial x^{4}}=0
$$

just barely suffice as a replacement [15].

In the case $j=0$ the missing field equations are replaced by the identities $\mathcal{F}_{r}=0$ themselves, which according to (64), i.e., $\frac{d \mathcal{F}_{r}}{d x^{4}}=0$, evolve in time.

We want to make one last observation with regard to the formula $\left(63^{\prime}\right)$. Let us inquire into the subgroup of our group that leaves all of the points of the slice $x^{4}=x_{0}^{4}$ invariant; this group is obviously a normal subgroup. The conditions

$$
\delta x^{\nu}=0 \text { for } x^{4}=x_{0}^{4}
$$

imply that

$$
\left(\xi^{r}\right)_{x^{4}=x_{0}^{4}}=\left(\frac{\partial \xi^{r}}{\partial x^{\sigma}}\right)_{x^{4}=x_{0}^{4}}=\ldots=\left[\frac{\partial^{k} \xi^{r}}{\partial x^{\sigma} \ldots \partial x^{\tau}}\right]_{x^{4}=x_{0}^{4}}=0 ;
$$

(in view of $\left(63^{\prime}\right)$ the infinitesimal transformation then reads

$$
\overline{\mathcal{S}}=\int d x^{1} d x^{2} d x^{3} \sum_{i=k+1}^{i=j}(-1)^{j-i} \frac{d^{j-i} \mathcal{F}_{r}}{\left(d x^{4}\right)^{j-i}} s_{i}{ }^{r}
$$

where $\frac{d^{j-i} \mathcal{F}_{r}}{\left(d x^{4}\right)^{j-i}}$ is taken at $x^{4}=x_{0}^{4}$ and the

$$
s_{i}^{r} \equiv\left[\frac{\partial^{i} \xi^{r}}{\left(\partial x^{4}\right)^{i}}\right]_{x^{4}=x_{0}^{4}}
$$

are arbitrary spatial functions [16]. The group $\overline{\mathcal{S}}$ is at every point of the slice a $r_{0}(j-k)$ parametric invariant subgroup. The group (52) considered in Section 6 is a subgroup of this group. 


\subsection{Covariance of the procedure under the group action}

Using the results obtained so far we are now in position to easily settle the question whether the procedure is covariant.

The formula (45) implies that for an arbitrary group transformation every functional $\Phi(Q, \mathcal{P})$ is subject to a similarity transformation of the form

$$
\Phi^{\prime}=S^{-1} \Phi S
$$

where according to (58) $S$ is time independent.

Furthermore, as is easy to see ${ }^{15}$ that formula (45) is also true for infinitesimal transformations $\overline{\mathcal{N}}$ of the group, i.e., it holds when all of the field quantities are subject to the infinitesimal transformation $\overline{\mathcal{M}}$,

$$
\omega \delta^{*} \overline{\mathcal{N}}=[\overline{\mathcal{M}}, \overline{\mathcal{N}}]
$$

hence, more generally,

$$
\overline{\mathcal{N}}^{\prime}=S^{-1} \overline{\mathcal{N}} S
$$

From (66) the covariance of the CR (37) follows immediately. According to (35) the Hamiltonian consists of only $Q$ and $\mathcal{P}$ dependent functional $\overline{\mathcal{H}_{0}}$ and a term $\overline{\lambda^{r} \mathcal{F}_{r}}$ that according to section 6 represents a special infinitesimal transformation $\overline{\mathcal{N}}$. On account of $(66)$ and $\left(67^{\prime}\right)$ the canonical field equations are also subject to a (constant in time) unitary transformation, under which, as is well known, they remain invariant.

All that remains to investigate is the variation of the left hand sides $\mathcal{F}_{r}$ of the identities (29). According to (67) they vary as

$$
\omega \delta^{*} \mathcal{F}_{r}=\left[\overline{\mathcal{M}}, \mathcal{F}_{r}\right]
$$

Thus it follows from the fact that the group $\overline{\mathcal{S}}$ defined in (65) is an invariant subgroup,

$$
\left[\overline{\mathcal{M}}, \mathcal{F}_{r}\right]=\sum_{i=k+1}^{i=j} \alpha_{i}^{r s} \frac{d^{j-i} \mathcal{F}_{s}}{\left(d x^{4}\right)^{j-i}}
$$

According to $(68)$ and $\left(68^{\prime}\right)$ we therefore have $\delta^{*} \mathcal{F}_{r}=0$, i.e., the proper identities $\mathcal{F}_{r}=$ 0 are invariant, due indeed to the identities themselves and possible time derivatives thereof.

15 If one replaces $\Phi$ in

$$
\Phi^{\prime}=\Phi+\frac{1}{\omega}[\overline{\mathcal{N}}, \Phi]
$$

by

$$
\tilde{\Phi}=\Phi+\frac{1}{\omega}[\overline{\mathcal{M}}, \Phi]
$$

and $\Phi^{\prime}$ by

$$
\tilde{\Phi}^{\prime}=\Phi^{\prime}+\frac{1}{\omega}\left[\overline{\mathcal{M}}, \Phi^{\prime}\right]
$$

then it follows after an easy calculation that

$$
\tilde{\Phi}^{\prime}=\tilde{\Phi}+\frac{1}{\omega}\left[\overline{\mathcal{N}}+\frac{1}{\omega}[\overline{\mathcal{M}}, \overline{\mathcal{N}}], \tilde{\Phi}\right]
$$

cf. also E. Noether, Gött. Nach. (1918), p. 252 [17]. 


\subsection{Extension of the theory to the "second case" of Section 1.1}

We indicate briefly how the theory above is extended to the "second case" defined in Section 1.1.

Our group would then have the simple form:

$$
\begin{aligned}
\bar{\delta} x^{\nu} & =0, \\
\bar{\delta} Q_{\alpha} & =c_{\alpha r} \xi^{r} .
\end{aligned}
$$

With

$$
\mathcal{L}^{\prime} \equiv \frac{d}{d x^{\nu}}\left[\underline{f^{\nu, \alpha \rho}(Q) Q_{\alpha, \rho}}\right]
$$

we have according to (12)

$$
\bar{\delta}\left(\mathcal{L}+\mathcal{L}^{\prime}\right)=0
$$

1. Let us next calculate $\bar{\delta} \mathcal{L}^{\prime}$. I maintain that $\bar{\delta} \mathcal{L}^{\prime}$ takes the form

$$
\bar{\delta} \mathcal{L}^{\prime}=\frac{d}{d x^{\nu}}\left(\underline{\mathcal{R}^{\alpha \nu} \bar{\delta} Q_{\alpha}}\right)
$$

or

$$
\bar{\delta} \mathcal{L}^{\prime}=\frac{d}{d x^{\nu}}\left(\mathcal{I}_{r}^{\nu} \xi^{r}\right)
$$

Because we obtain first

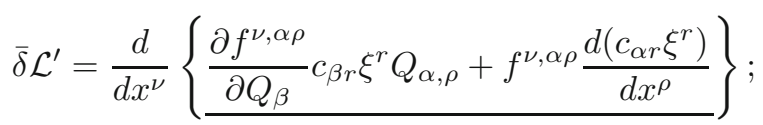

if we set

$$
r^{\alpha \nu}=-\frac{d f^{\nu, \alpha \rho}}{d x^{\rho}}+Q_{\beta, \rho} \frac{\partial f^{\nu, \beta \rho}}{\partial Q_{\alpha}}
$$

and

$$
\mathcal{I}_{r}{ }^{\nu}=\underline{r^{\alpha \nu} c_{\alpha r}}
$$

so we have

$$
\bar{\delta} \mathcal{L}^{\prime}=\frac{d}{d x^{\nu}}\left\{\mathcal{I}_{r}{ }^{\nu} \xi^{r}+\frac{d}{d x^{\rho}}\left(f^{\nu, \alpha \rho} c_{\alpha r} \xi^{r}\right)\right\} .
$$

Now we use (70) and write out the requirement that the coefficients of the second derivatives of the $\xi^{r}$ vanish identically. Since $\mathcal{L}$ contains no second derivatives of the $\xi^{r}$, we have according to $(75)$

$$
\left(f^{\nu, \alpha \rho}+f^{\rho, \alpha \nu}\right) c_{\alpha r} \equiv 0 .
$$

As a consequence (75) is reduced to

$$
\bar{\delta} \mathcal{L}^{\prime}=\frac{d}{d x^{\nu}}\left(\mathcal{I}_{r}{ }^{\nu} \xi^{r}\right)
$$

Now we set

$$
\mathcal{R}^{\alpha \nu}=\underline{r^{\alpha \nu}}
$$

and notice that instead of (74) we can also write

$$
\mathcal{I}_{r}{ }^{\nu}=\underline{\mathcal{R}^{\alpha \nu} c_{\alpha r}},
$$

thus we have proven formulas (71) and (72). 
2. Now we set up the analogues of the identities (28) that in the first case contain the proper identities (29). For that purpose we must simply set the coefficients of the $\frac{d \xi^{r}}{d x^{\nu}}$ in $(70)$ equal to zero. We obtain

$$
\underline{\left(\mathcal{P}^{\alpha \nu}+\mathcal{R}^{\alpha \nu}\right) c_{\alpha r}}=0
$$

In particular for $\nu=4$ :

$$
\underline{\left(\mathcal{P}^{\alpha}+\mathcal{R}^{\alpha 4}\right) c_{\alpha r}}=0
$$

or, once again substituting

$$
\begin{gathered}
\mathcal{F}_{r}=\frac{\mathcal{P}^{\alpha} c_{\alpha r}}{} \text { and } \mathcal{I}_{r}{ }^{4}=\mathcal{I}_{r}, \\
\mathcal{F}_{r}+\mathcal{I}_{r}=0 .
\end{gathered}
$$

3. The identities (79) are proper, i.e., we have

$$
\frac{\partial \mathcal{I}_{r}}{\partial \dot{Q}_{\alpha}}=0
$$

Instead of directly proving (80) we will prove more generally that

$$
\frac{\partial\left(r^{\beta \rho} c_{\beta r}\right)}{\partial Q_{\alpha, \nu}}=-\frac{\partial\left(r^{\beta \nu} c_{\beta r}\right)}{\partial Q_{\alpha, \rho}},
$$

from which (80) follows via (74) when $\nu=\rho=4$.

For this purpose we set equal to zero the coefficient of $\xi^{r}$ in (70): it is a term linear in the second derivative $Q_{\alpha, \rho \nu}$, with coefficients that only depend on $Q$. Since this term vanishes identically for arbitrary $Q_{\alpha, \rho \nu}$, we can in particular assign $c$-number values to the $Q_{\alpha, \rho \nu}$ and then separately set to zero the coefficients of the $Q_{\alpha, \rho \nu}$. Using the formula that is valid for arbitrary $\mathcal{K}^{\rho}\left(Q_{\alpha} ; Q_{\alpha, \nu}\right)$ :

$$
\frac{\partial}{\partial Q_{\alpha, \nu}} \frac{d}{d x^{\rho}} \mathcal{K}^{\rho}\left(Q_{\alpha} ; Q_{\alpha, \nu}\right)=\frac{\partial \mathcal{K}^{\nu}}{\partial Q_{\alpha}}+\frac{d}{d x^{\rho}} \frac{\partial \mathcal{K}^{\rho}}{\partial Q_{\alpha, \nu}},
$$

we find for these coefficients according to (71 and (73) [18]

$$
\frac{\partial\left(r^{\beta \rho} c_{\beta r}\right)}{\partial Q_{\alpha, \nu}}+\frac{\partial\left(r^{\beta \nu} c_{\beta r}\right)}{\partial Q_{\alpha, \rho}}
$$

setting this expression equal to zero gives $\left(80^{\prime}\right)$.

By the way, according to (81) it follows furthermore from (73) that

$$
\frac{\partial r^{\alpha \nu}}{\partial Q_{\beta, \rho}}=\frac{\partial f^{\nu, \beta \rho}}{\partial Q_{\alpha}}-\frac{\partial f^{\nu, \alpha \rho}}{\partial Q_{\beta}}=\frac{\partial \mathcal{R}^{\alpha \nu}}{\partial Q_{\beta, \rho}} ;
$$

therefore instead of $\left(80^{\prime}\right)$ we can write

$$
\frac{\partial\left(\mathcal{R}^{\beta \rho} c_{\beta r}\right)}{\partial Q_{\alpha, \nu}}=-\frac{\partial\left(\mathcal{R}^{\beta \nu} c_{\beta r}\right)}{\partial Q_{\alpha, \rho}} .
$$

4. The calculations of Sections 1.3 and 1.4 can be applied word for word to the present case with $\mathcal{P}^{\alpha}+\mathcal{R}^{\alpha 4}$ taking the role of $\mathcal{P}^{\alpha}[19]$. 
The derived expression $\overline{\mathcal{M}}$ for the infinitesimal transformation, as derived in Section 1.5, undergoes an analogous modification since $\mathcal{P}^{\alpha \nu}$ is no longer a tensor density ${ }^{16}$.

Rather, we have according to (50) and (70)

$$
\bar{\delta} \mathcal{P}^{\alpha \nu} \equiv-\underline{-\mathcal{P}^{\beta \nu} \frac{\partial \bar{\delta} Q_{\beta}}{\partial Q_{\alpha}}}-\frac{\partial\left(\bar{\delta} \mathcal{L}^{\prime}\right)}{\partial Q_{\alpha, \nu}} ;
$$

but by (71), referring to (81),

$$
\frac{\partial\left(\bar{\delta} \mathcal{L}^{\prime}\right)}{\partial Q_{\alpha, \nu}} \equiv \frac{\partial\left(\mathcal{R}^{\beta \nu} \bar{\delta} Q_{\beta}\right)}{\partial Q_{\alpha}}+\frac{d}{d x^{\rho}} \frac{\partial\left(\mathcal{R}^{\beta \rho} \bar{\delta} Q_{\beta}\right)}{\partial Q_{\alpha, \nu}},
$$

i.e., taking (83) into account

$$
\bar{\delta} \mathcal{P}^{\alpha \nu}=-\mathcal{P}^{\beta \nu} \frac{\partial \bar{\delta} Q_{\beta}}{\partial Q_{\alpha}}-\frac{\partial\left(\mathcal{R}^{\beta \nu} \bar{\delta} Q_{\beta}\right)}{\partial Q_{\alpha}}+\frac{d}{d x^{\rho}} \frac{\partial\left(\mathcal{R}^{\beta \nu} \bar{\delta} Q_{\beta}\right)}{\partial Q_{\alpha, \rho}} .
$$

In particular due to $(80)$, for $\nu=4$ this becomes [20]

$$
\bar{\delta} \mathcal{P}^{\alpha}=-\mathcal{P}^{\beta} \frac{\partial \bar{\delta} Q_{\beta}}{\partial Q_{\alpha}}-\frac{\partial\left(\mathcal{R}^{\beta 4} \bar{\delta} Q_{\beta}\right)}{\partial Q_{\alpha}}+\frac{d}{d x^{\bar{\rho}}} \frac{\partial\left(\mathcal{R}^{\beta 4} \bar{\delta} Q_{\beta}\right)}{\partial Q_{\alpha, \bar{\rho}}},
$$

i.e.,

with

$$
\omega \bar{\delta} \mathcal{P}^{\alpha}=\left[\overline{\mathcal{N}}, \mathcal{P}^{\alpha}\right]
$$

$$
\mathcal{N}=\underline{\left(\mathcal{P}^{\alpha}+\mathcal{R}^{\alpha 4}\right) \bar{\delta} Q_{\alpha}}
$$

Once again on account of (80) we also have

$$
\omega \bar{\delta} Q_{\alpha}=\left[\overline{\mathcal{N}}, Q_{\alpha}\right],
$$

so that we have in $\overline{\mathcal{N}}$ the desired extension of $\overline{\mathcal{M}}$.

From expression (86) it follows exactly as in Section 1.6 that the left hand sides $\mathcal{F}_{r}+\mathcal{I}_{r}$ of the proper identities commute as a consequence of the identities themselves.

The considerations of Section 1.7 concerning the constancy in time of the $\overline{\mathcal{M}}$ as well as the proof of covariance of Section 1.8 can be carried over without change to $\overline{\mathcal{N}}$. In particular, since it is assumed here that $j=0$, the identities $\mathcal{F}_{r}+\mathcal{I}_{r}=0$ play the role of the missing field equations.

\subsection{Observations concerning the simultaneous treatment of multiple groups}

In case the Lagrangian admits several groups the above theory is still applicable considering that the infinitesimal transformation of the direct product of the relevant groups consists of the sum of infinitesimal transformations of the individual groups. In particular the $\mathcal{F}_{r}$ of each individual group commute not only with each other (due to $\mathcal{F}_{r}=0$ ), but also with the $\mathcal{F}_{r}$ belonging to other groups. It is also permissible that "case 1 " ( $\mathcal{L}$ is a density ) may apply to some groups, and "case 2 " treated in Section 9 may apply to others. For the latter case we must simply replace the $\mathcal{F}_{r}$ by $\mathcal{F}_{r}+\mathcal{I}_{r}$; these once again commute not only among themselves but also with the remaining $\mathcal{F}_{r}$.

It follows from this observation that one may treat independently the individual groups admitted by the Lagrangian.

\footnotetext{
16 Although neither $\mathcal{P}^{\alpha} \nu$ nor $\mathcal{R}^{\alpha \nu}$ are tensor densities, it is easy to show that $\mathcal{P}^{\alpha \nu}+\mathcal{R}^{\alpha \nu}$ is a tensor density.
} 


\section{Part two: Applications}

\subsection{The Lagrange function}

We wish to construct a Lagrangian that includes not only electromagnetic and material fields, but also the gravitational field. Concerning the latter, we will adopt the one-body theory proposed by Fock ${ }^{17}$ and Weyl ${ }^{18}$ : we describe the gravitational field through the introduction at every point of four orthogonal vectors $h_{i, \nu}(i=1,2,3,4)$ and we demand that the laws of nature be covariant under $x$-dependent Lorentz transformation of the "Vierbeine". This covariance, which we want to call "genuine bein covariance" following Levi Civita ${ }^{19}$ is fundamentally different from the "local bein covariance" demanded by the Einsteinian theory of Fernparallelismus in which all of the tetrads are rigidly linked (constant Lorentz transformations of the tetrads). In agreement with Fock (and contrary to Weyl) we describe the material field through a four-component wave function $\psi \equiv\left(\psi_{1}, \psi_{2}, \psi_{3}, \psi_{4}\right)$. For the electromagnetic field we select as variables the components $\phi_{\mu}$ of the four-potential ${ }^{20}$.

The Lagrangian is composed additively of three parts that correspond to the three designated fields (and simultaneously contain the field interactions).

Letting

$$
E_{\mu \nu}=\frac{\partial \phi_{\nu}}{\partial x^{\mu}}-\frac{\partial \phi_{\mu}}{\partial x^{\nu}}
$$

represent the electromagnetic field tensor, then the radiation term in the Lagrangian is

$$
\mathcal{E}=\frac{1}{4} E_{\mu \nu} \mathcal{E}^{\mu \nu}
$$

with

$$
\mathcal{E}^{\mu \nu}=E^{\mu \nu} h^{\prime}
$$

where $h^{\prime}$ is the determinant of $h_{i, \nu}$ and the $E^{\mu \nu}$ are the contravariant components of the tensor $E_{\mu \nu}$.

In order to specify the matter term we choose a specific system of Dirac matrices. Let us start from the Pauli matrices ${ }^{21}$

$$
\rho_{1}=\left(\begin{array}{cc}
i & 0 \\
0 & -i
\end{array}\right), \rho_{2}=\left(\begin{array}{ll}
0 & i \\
i & 0
\end{array}\right), \rho_{3}=\left(\begin{array}{cc}
0 & -1 \\
1 & 0
\end{array}\right) .
$$

Thus let us set

$$
\left\{\begin{array}{c}
\alpha_{\bar{l}}=-i\left(\begin{array}{cc}
\rho_{\bar{l}} & 0 \\
0 & -\rho_{\bar{l}}
\end{array}\right)(\bar{l}=1,2,3), \\
\alpha_{4}=1 .
\end{array}\right.
$$

We now introduce the notation

$$
e_{\bar{k}}=-1, \quad e_{4}=1,
$$

so the matrices $\alpha_{i}$ are Hermitian and they have the commutation property

$$
\alpha_{m} \alpha_{k} e_{k}+\alpha_{k} \alpha_{m} e_{m}=2 e_{m} \delta_{m k} .
$$

\footnotetext{
17 V. Fock, Zeit. F. Phys. 57, 261 (1930).

18 H. Weyl, Zeit. F. Phys. 56, 330 (1929).

19 Berliner Berichte 1929, p. 137.

${ }^{20}$ Since we have set $x^{4}=c t$ we have $\phi_{4}=-\phi$, where $\phi$ represents the scalar potential.

21 This differs from the Fock matrices (loc. cit.) only slightly. The essentially different feature of the specialization is $\sigma_{4}=1$ (In Fock's notation $\sigma_{0}=1$ ).
} 
Furthermore we need the matrix

$$
\sigma=\left(\begin{array}{ll}
0 & 1 \\
1 & 0
\end{array}\right)
$$

(where the ones represent two-row identity matrices).

With regard to the latin indices a sum over doubly repeated indices is understood, whereby the factor $e_{k}$ is to be ignored when counting the number of indices. In addition to the $h_{i, \nu}$ the contravariant $h_{i}{ }^{\nu}$ also appear, and they satisfy the relations [21]

$$
\left\{\begin{array}{l}
h_{k}^{\nu} h_{l, \nu}=e_{k} \delta_{k l} \\
e_{k} h_{k}^{\nu} h_{k, \mu}=\delta_{\mu}^{\nu}
\end{array}\right.
$$

which express the orthogonality of the tetrads in the space with metric

$$
g_{\mu \nu}=e_{k} h_{k, \mu} h_{k, \nu} .
$$

Defining

$$
\eta_{\rho \sigma}^{l}=\frac{\partial h_{l, \rho}}{\partial x^{\sigma}}-\frac{\partial h_{l, \sigma}}{\partial x^{\rho}}
$$

and

$$
2 \gamma_{m k l}=\underline{\left(\eta_{\rho \sigma}^{l} h_{m}^{\sigma} h_{k}^{\rho}+\eta_{\rho \sigma}^{m} h_{l}^{\sigma} h_{k}^{\rho}+\eta_{\rho \sigma}^{k} h_{m}^{\sigma} h_{l}^{\rho}\right) h^{\prime}},
$$

and

$$
C_{l}=\frac{1}{4} e_{k} \alpha_{m} \alpha_{k} \gamma_{m k l}+\frac{e}{\omega} \phi_{\sigma} h_{l}^{\sigma} h^{\prime}, \quad\left(\omega=\frac{h c}{2 \pi i}\right),
$$

and finally

$$
\gamma^{\sigma}=e_{k} \alpha_{k} h_{k}^{\sigma} h^{\prime}
$$

then the matter term in the Lagrangian reads

$$
\mathbf{R} \omega \psi^{*}\left(\gamma^{\sigma} \frac{\partial \psi}{\partial x^{\sigma}}-e_{l} \alpha_{l} C_{l} \psi\right)-m c^{2} \psi^{*} \sigma \psi h^{\prime} .
$$

( $x^{*}$ is the complex conjugate of $x . \mathbf{R} x$ is the real part of $x . \mathbf{I} x$ is the imaginary part of $x$.)

Now we have (cf. Fock, loc. cit., formula (24)),

$$
e_{l}\left(\alpha_{l} C_{l}+C_{l}^{\dagger} \alpha_{l}\right)=-\frac{\partial \gamma^{\sigma}}{\partial x^{\sigma}}
$$

and consequently

$$
\mathbf{I} \omega \psi^{*}\left(\gamma^{\sigma} \frac{\partial \psi}{\partial x^{\sigma}}-e_{l} \alpha_{l} C_{l} \psi\right)=\frac{\omega}{2} \frac{\partial}{\partial x^{\sigma}}\left(\psi^{*} \gamma^{\sigma} \psi\right) .
$$

We can therefore take as the matter term

$$
\mathcal{W}=\omega \psi^{*}\left(\gamma^{\sigma} \frac{\partial \psi}{\partial x^{\sigma}}-e_{l} \alpha_{l} C_{l} \psi\right)-m c^{2} \psi^{*} \sigma \psi h^{\prime}
$$

instead of (101).

For the gravitational part we take $\frac{1}{2 \kappa} \mathcal{G}$, where $\kappa=\frac{8 \pi f}{c^{4}}(f=$ Newton's gravitational constant) and

$$
\mathcal{G}=e_{k} e_{l} \eta_{\rho \sigma}^{l} h_{l}^{\rho} h_{k}^{\rho^{\prime}} g^{\sigma \sigma^{\prime}} h^{\prime} \eta_{\rho^{\prime} \sigma^{\prime}}^{k}-\frac{1}{2} e_{k} e_{l} \eta_{\rho \sigma}^{l} h_{l}^{\rho^{\prime}} h_{k}^{\rho} g^{\sigma \sigma^{\prime}} h^{\prime} \eta_{\rho^{\prime} \sigma^{\prime}}^{k}-\frac{1}{4} e_{l} \eta_{\rho \sigma}^{l} g^{\sigma \sigma^{\prime}} g^{\rho \rho^{\prime}} h^{\prime} \eta_{\rho^{\prime} \sigma^{\prime}}^{l}
$$


as is easily checked (cf. e.g. Weyl, loc. cit.) that $\mathcal{G}$ differs from the scalar curvature density $\mathcal{R}$ by a divergence

$$
\mathcal{R}=\mathcal{G}-2 \frac{d}{d x^{\nu}} \underline{\left(e_{l} h_{l}^{\nu} \frac{\partial\left(h_{l}^{\sigma} h^{\prime}\right)}{\partial x^{\sigma}}\right)} .
$$

Altogether we have therefore

$$
\mathcal{L}=\frac{1}{2 \kappa} \mathcal{G}+\mathcal{E}+\mathcal{W}
$$

In contrast to the usual form of relativity theory where the field quantities $g_{\mu \nu}$ were not vectors but were rather second rank tensors, the two constituent parts $\mathcal{G}$ and $2 \frac{d}{d x^{\nu}}\left(e_{l} h_{l}^{\nu} \frac{\partial\left(h_{l}^{\sigma} h^{\prime}\right)}{\partial x^{\sigma}}\right)$ of $\mathcal{R}$ in (105) are scalar densities under the general relativistic transformation group. On the other hand $\mathcal{G}$ is not by itself bein invariant, but $\mathcal{R}$ is.

\subsection{The gauge invariance group}

The simplest group admitted by our function $\mathcal{L}$ is the gauge invariance group for which the $x^{\nu}$ and the $h_{l, \nu}$ remain invariant while the $\phi_{\nu}$ and $\psi$ transform as follows [22]

$$
\left\{\begin{array}{c}
\delta \phi_{\nu}=\frac{\partial \xi}{\partial x^{\nu}} \\
\delta \psi=-\frac{e}{\omega} \xi \psi
\end{array}\right.
$$

Under this group $\delta \mathcal{L}=0$.

In order to ease the comparison with the general theory we $\operatorname{set}^{22}$

$$
\phi_{\nu}=Q_{\nu}, \psi=Q_{5}
$$

so that we have

$$
c_{\nu}^{\mu}=\delta_{\nu}^{\mu}, c_{5}^{\mu}=0
$$

with, hence, the single resulting identity

$$
\mathcal{P}^{4}=0
$$

This follows directly from the explicit calculation of the $\mathcal{P}^{\alpha \nu}$ :

$$
\left\{\begin{array}{c}
\mathcal{P}^{\mu \nu}=\mathcal{E}^{\nu \mu} \\
\mathcal{P}^{5 \nu}=\omega \psi^{*} \gamma^{\nu}
\end{array}\right.
$$

In order to discuss this simple example further we first disregard gravitation, i.e., we set $h_{i, \nu}=\delta_{i, \nu}[23]$.

The Hamiltonian then takes the form

$$
\mathcal{H}=\mathcal{H}_{0}+\lambda \mathcal{P}^{4}
$$

where $\mathcal{H}_{0}$ is for example the special Hamiltonian function selected in H.P.II that does not contain $\mathcal{P}^{4}$.

\footnotetext{
${ }^{22}$ Since the $\psi$ are not Hermitian it is necessary to make some small modifications in order to adapt these variables to the formalism. There is no need to go into these details here.
} 
The field equations read

$$
\begin{gathered}
\left\{\begin{array}{c}
\omega \dot{Q}_{\bar{\nu}}=\left[\overline{\mathcal{H}}_{0}, Q_{\bar{\nu}}\right], \\
\dot{Q}_{4}=\lambda, \\
\omega \dot{Q}_{5}=\left[\overline{\mathcal{H}}_{0}, Q_{5}\right] ;
\end{array}\right. \\
\omega \dot{\mathcal{P}}^{\alpha}=\left[\overline{\mathcal{H}}_{0}, \mathcal{P}^{\alpha}\right], \quad(\alpha=1, \ldots, 5) .
\end{gathered}
$$

Since we also have $j=1$ we have a constraint ${ }^{23}$ besides (108)

$$
\left[\overline{\mathcal{H}}_{0}, \mathcal{P}^{4}\right]=0
$$

So $\lambda$ in (111) remains completely undetermined and the fourth equation (112) is replaced by (113).

The infinitesimal transformation $\overline{\mathcal{M}}$ reads here:

$$
\overline{\mathcal{M}}=\int\left\{\frac{\partial \xi}{\partial x^{\nu}} \mathcal{E}^{4 \nu}-e \xi \psi^{*} \gamma^{4} \psi\right\} d x^{1} d x^{2} d x^{3},
$$

or through integration by parts

$$
\overline{\mathcal{M}}=\int\left\{\frac{\partial \xi}{\partial x^{4}} \mathcal{P}^{4}-\xi\left[\frac{\partial \mathcal{E}^{4 \bar{\nu}}}{\partial x^{\bar{\nu}}}+e \psi^{*} \gamma^{4} \psi\right]\right\} d x^{1} d x^{2} d x^{3}
$$

The square bracket is nothing other than $\frac{1}{\omega}\left[\overline{\mathcal{H}}_{0}, \mathcal{P}^{4}\right]$, or $\dot{\mathcal{P}}^{4}$, so that

$$
\overline{\mathcal{M}}=\int\left\{\frac{\partial \xi}{\partial x^{4}} \mathcal{P}^{4}-\xi \frac{d \mathcal{P}^{4}}{d x^{4}}\right\} d x^{1} d x^{2} d x^{3},
$$

in agreement with $\left(63^{\prime}\right)$.

According to the general theory $\mathcal{P}^{4}=0$ must hold due to the field equations and the identities; this is in fact the continuity equation of electricity.

\subsection{General relativistic covariance}

For an arbitrary coordinate transformation

$$
\delta x^{\nu}=\xi^{\nu},
$$

we have

$$
\delta h_{l, \nu}=-h_{l, \mu} \frac{\partial \xi^{\mu}}{\partial x^{\nu}}
$$

and, furthermore,

$$
\left\{\begin{array}{c}
\delta \phi_{\nu}=-\phi_{\mu} \frac{\partial \xi^{\mu}}{\partial x^{\nu}} \\
\delta \psi=0
\end{array}\right.
$$

The Lagrangian behaves as a scalar density under this transformation.

We retain the notation of the previous paragraphs for the momenta conjugate to $\phi_{\nu}$ and $\psi$, and we represent the momenta $\mathcal{P}^{\alpha \mu}$ conjugate to the $h_{i, \mu}$ by $\mathcal{P}_{i}^{\nu \mu}$ so the inproper identities (28) read in the present case

$$
\underline{\phi_{\rho}\left(\mathcal{E}^{\mu \nu}+\mathcal{E}^{\nu \mu}\right)+h_{i, \rho}\left(\mathcal{P}_{i}^{\nu \mu}+\mathcal{P}_{i}^{\mu \nu}\right)}=0
$$

23 In the notation of H.P.II (113) reads $C=0$. 
taking into account that $\mathcal{E}^{\mu \nu}+\mathcal{E}^{\nu \mu}=0$ they reduce to

$$
\mathcal{P}_{i}^{\nu \mu}+\mathcal{P}_{i}^{\mu \nu}=0
$$

and have the four proper identities

$$
\mathcal{P}_{l}^{4}=0
$$

as a consequence.

The straightforward calculation in fact gives (116) since $\mathcal{G}$ and $\mathcal{W}$ depend on the $h_{i, \nu, \mu}$ only through the $\eta_{\tau \sigma}^{i}$ and

$$
\frac{\partial \eta_{\rho \sigma}^{i}}{\partial h_{i, \nu, \mu}}=\delta_{\rho}^{\mu} \delta_{\sigma}^{\nu}-\delta_{\rho}^{\nu} \delta_{\sigma}^{\mu}
$$

i.e., is antisymmetric in $\mu$ and $\nu$. One finds

$$
\left\{\begin{array}{c}
\mathcal{P}_{i}^{\nu \mu}=\frac{\left[\frac{\eta_{\rho \sigma}^{i} g^{\nu \sigma} g^{\mu \rho}+2 e_{l} \eta_{\rho \sigma}^{l} h_{l}^{\rho}\left(g^{\sigma \mu} h_{i}^{\nu}-g^{\sigma \nu} h_{i}^{\mu}\right)}{\left.-\frac{e_{l} \eta_{\rho \sigma}^{l} h_{i}^{\rho}\left(h_{l}^{\nu} g^{\sigma \mu}-h_{l}^{\mu} g^{\sigma \nu}\right) e_{i} h^{\prime}}{2 \kappa}\right] \frac{1}{2 \kappa}}\right.}{-\mathbf{R} \frac{\omega}{4} \psi^{*} \alpha_{l} \alpha_{m} \alpha_{k} \psi e_{l} e_{k} \frac{\partial \gamma_{m k l}}{\partial h_{i, \nu, \mu}}},
\end{array}\right.
$$

The infinitesimal transformation $\overline{\mathcal{M}}$ takes the form

$$
\overline{\mathcal{M}}=-\int d x^{1} d x^{2} d x^{3}\left\{\frac{\partial \xi^{\mu}}{\partial x^{4}}\left(h_{i, \mu} \mathcal{P}_{i}^{4}+\phi_{\mu} \mathcal{P}^{4}\right)-\xi^{\mu}\left[\frac{\partial}{\partial x^{\bar{\nu}}} \underline{\left(h_{i, \mu} \mathcal{P}_{i}^{\bar{\nu}}+\phi_{\mu} \mathcal{P}^{\bar{\nu}}\right)}+\mathcal{G}_{\mu}\right]\right\}
$$

let us consider in particular the translation $\xi^{\mu}=\epsilon^{\mu}=$ const so that $\frac{d \overline{\mathcal{M}}}{d x^{4}}=0$ yields the energy-momentum conservation law

$$
\overline{\mathcal{G}}_{\mu}=\text { const. }
$$

for a linear transformation we derive from $\overline{\mathcal{M}}$ the generalization of the angular momentum conservation law (cf. H.P.II, p. 177).

According to the general theory (Sect. 1.7) the setting of the coefficients of $\xi^{\mu}$ equal to zero yields a constraint

$$
\mathcal{G}_{\mu}+\frac{\partial}{\partial x^{\bar{\nu}}} \underline{\left(h_{i, \mu} \mathcal{P}_{i}^{\bar{\nu}}+\phi_{\mu} \mathcal{P}^{\bar{\nu}}\right)}=0
$$

F. Klein ${ }^{24}$ has already observed in another context that (119) is equivalent to four field equations [25].

\subsection{The true bein covariance}

For this group we have [26]

$$
\begin{gathered}
\delta x^{\nu}=0, \delta \phi_{\mu}=0, \\
\delta h_{i, \nu}=e_{k} \xi_{i k} h_{k, \nu}, \quad\left(\xi_{i k}=-\xi_{k i}\right),
\end{gathered}
$$

and based on (120) one easily shows that

$$
\delta \psi=\frac{1}{4} e_{k} \xi_{i k} \alpha_{i} \alpha_{k} \psi
$$

${ }^{24}$ Gött. Nach. 1918, p. 185. 
We have here an example of the "second case" treated in Section 1.9, since $\mathcal{G}$ is only locally invariant and only $\mathcal{W}$ is truly bein invariant; $\mathcal{M}$ and $\mathcal{E}$ are also true bein invariants.

For the purpose of calculating formula $(74), \mathcal{I}_{r}^{\mu} \equiv \mathcal{I}_{(i k)}^{\mu}$, it will be most useful to temporarily select as a variable

$$
Q_{l}^{\alpha} \equiv h^{\prime} h_{l}^{\alpha}
$$

Then according to (105)

$$
f_{l}^{\nu, \alpha \rho}=-\frac{1}{\kappa} e_{l} h_{l}^{\nu} \delta_{\rho}^{\alpha}
$$

and according to (120)

$$
c_{l, \alpha(i k)}=\delta_{i l} e_{k} h_{k}^{\alpha} h^{\prime}-\delta_{k l} e_{i} h_{i}^{\alpha} h^{\prime} .
$$

Referring now to (71) one easily finds that

$$
\mathcal{I}_{(i k)}^{\nu}=\frac{1}{\kappa} e_{i} e_{k} \frac{d}{d x^{\rho}}\left(h^{\prime} h_{i}^{\nu} h_{k}^{\rho}-h^{\prime} h_{i}^{\rho} h_{k}^{\nu}\right)
$$

To calculate $\mathcal{F}_{r} \equiv \mathcal{F}_{(i k)}$ it will be more convenient to return to the original variables $Q_{l, \alpha} \equiv h_{l, \alpha}$ and $Q_{5}=\psi$. Then we have to set

$$
c_{l, \alpha(i k)}=\delta_{i l} e_{k} h_{k, \alpha}-\delta_{k l} e_{i} h_{i, \alpha}
$$

and in addition, by $\left(120^{\prime}\right)$, we set

$$
c_{5(i k)}=\frac{1}{4}\left(e_{k} \alpha_{i} \alpha_{k} \psi-e_{i} \alpha_{k} \alpha_{i} \psi\right) .
$$

We have accordingly

$$
\mathcal{F}_{(i k)}=\mathcal{P}_{i}^{\nu} e_{k} h_{k, \nu}-\mathcal{P}_{k}^{\nu} e_{i} h_{i, \nu}+\frac{\omega}{4} e_{k} e_{l} h^{\prime} h_{l}^{4} \psi^{*}\left(\alpha_{l} \alpha_{i} \alpha_{k}-\alpha_{l} \alpha_{k} \alpha_{i}\right) \psi
$$

Now according to $(98)$

$$
\frac{\partial \gamma_{m j l}}{\partial h_{i, \nu, 4}} e_{k} h_{k, \nu}-\frac{\partial \gamma_{m j l}}{\partial h_{k, \nu, 4}} e_{i} h_{i, \nu}=h^{\prime} h_{l}^{4}\left(\delta_{i m} \delta_{k j}-\delta_{i j} \delta_{k m}\right) ;
$$

then according to (118) we set

$$
\mathcal{P}_{i}^{\nu}=\tilde{\mathcal{P}}_{i}^{\nu}-\mathbf{R} \frac{\omega}{4} e_{l} \psi^{*} \alpha_{l} \alpha_{m} \alpha_{j} \psi e_{j} \frac{\partial \gamma_{m j l}}{h_{i, \nu, 4}},
$$

where $\tilde{\mathcal{P}}_{i}^{\nu}$ represents the momentum in the absence of matter. Then the $\mathcal{F}_{(i k)}$ simplifies, as it must, to

$$
\mathcal{F}_{(i k)}=\underline{\tilde{\mathcal{P}}_{i}^{\nu} e_{k} h_{k, \nu}-\tilde{\mathcal{P}}_{k}^{\nu} e_{i} h_{i, \nu}}
$$

According to (121) and (123) the six proper identities read

$$
\underline{\tilde{\mathcal{P}}_{i}^{\nu} e_{k} h_{k, \nu}-\tilde{\mathcal{P}}_{k}^{\nu} e_{i} h_{i, \nu}}+\frac{1}{\kappa} e_{i} e_{k} \frac{d}{d x^{\rho}}\left(h^{\prime} h_{i}^{4} h_{k}^{\rho}-h^{\prime} h_{i}^{\rho} h_{k}^{4}\right)=0,
$$

which can also be obtained directly from (118). 


\subsection{Supplementary observations on the gravitational and matter fields}

1. After having sketched in the previous paragraphs how the Fock-Weyl one-body theory can be quantized, we would like to briefly address a point that is treated differently by Fock and by Weyl, namely the construction of the energy-momentum tensor $\mathcal{T}_{i}{ }^{\nu}$ of matter. The Fock approach leads to a non-symmetric tensor and seems for us to be inappropriate. We prefer the Weyl definition

$$
\mathcal{T}_{i}{ }^{\nu}=\frac{\delta \mathcal{W}}{\delta h_{i, \nu}}
$$

that because of the field equations gives a symmetric tensor. However, since Weyl works with a two-component $\psi$ while we want to stay with Fock's four-component theory, it would not be redundant to repeat here mutatis mutandis the Weyl calculation of $\mathcal{T}_{i}{ }^{\nu}$.

The symmetry of $\mathcal{T}_{i}{ }^{\nu}$ follows immediately from $\delta \mathcal{W}=0$, where $\delta$ is the variation (120), (120'), for it follows from setting to zero the coefficients of $\xi_{i k}$ that

$$
\mathcal{T}_{i}{ }^{\nu} e_{k} h_{k, \nu}-\mathcal{T}_{k}{ }^{\nu} e_{i} h_{i, \nu}=-\frac{1}{2} \mathbf{R} \frac{\delta \mathcal{W}}{\delta \psi}\left(e_{k} \alpha_{i} \alpha_{k}-e_{i} \alpha_{k} \alpha_{i}\right) \psi,
$$

i.e.,

$$
\mathcal{T}_{i}^{\nu} e_{k} h_{k, \nu}-\mathcal{T}_{k}{ }^{\nu} e_{i} h_{i, \nu}=0
$$

using the field equations

$$
\frac{\delta \mathcal{W}}{\delta \psi}=0 \text { and } \frac{\delta \mathcal{W}}{\delta \psi^{*}}=0
$$

This equation expresses the fact that the tensor [28]

$$
\mathcal{T}_{i k}^{\prime \prime}=e_{i} e_{k} \mathcal{T}_{i}^{\nu} h_{k, \nu}
$$

is symmetric with respect to $i$ and $k$.

Instead of $(125 c)$ we can just as well set

$$
\mathcal{T}_{i}{ }^{\nu}=\frac{\delta \mathbf{R} \mathcal{W}}{\delta h_{i, \nu}}
$$

which will give a real-valued tensor $\mathcal{T}_{i}{ }^{\nu}$. It is more convenient to calculate

$$
\mathcal{T}_{i, \nu}^{\prime}=\frac{\delta \mathbf{R} \mathcal{W}}{\delta h_{i}^{\nu}}=-e_{k} h_{k, \nu} h_{k, \rho} \mathcal{T}_{k}^{\rho} \equiv e_{i} h^{\prime} T_{i, \nu}^{\prime}
$$

Based on (103) we find

$$
\left\{\begin{aligned}
T_{i, \nu}^{\prime}= & \mathbf{R} \omega \psi^{*} \alpha_{i} \frac{\partial \psi}{\partial x^{\nu}}-e \psi^{*} \alpha_{i} \psi \phi_{\nu}-h_{i, \nu} W \\
& +\mathbf{R} \frac{\omega}{4} e_{k} h_{k}^{\rho} h_{m, \nu} \frac{\partial}{\partial x^{\rho}}\left\{\psi^{*} \alpha_{i} \alpha_{m} \alpha_{k} \psi\right\} \\
& -\mathbf{R} \frac{\omega}{4} e_{i} e_{l} e_{k} \psi^{*} \alpha_{l} \alpha_{m} \alpha_{k} \psi\left\{\frac{\partial \gamma_{m k l}}{\partial h_{i}^{\nu}}-\frac{\partial}{\partial x^{\rho}} \frac{\partial \gamma_{m k l}}{\partial h_{i, \rho}^{\nu}}\right\} \\
\text { with } W= & \frac{1}{h^{\prime}} \mathcal{W} .
\end{aligned}\right.
$$

We confine ourselves now to special relativity in setting

$$
h_{i}^{\nu}=e_{i} h_{i, \nu}=\delta_{i \nu}
$$


Then (128c) becomes ${ }^{25}$

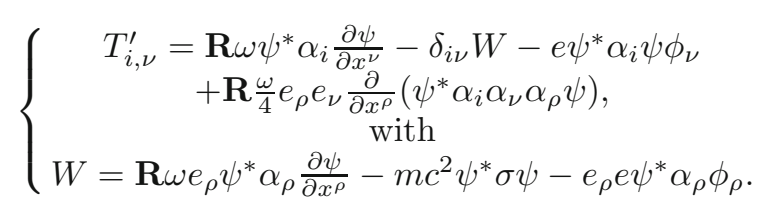

In particular we then have [29]

$$
T_{44}^{\prime}=\mathbf{R} \omega \psi^{*} \alpha_{\bar{\rho}} \frac{\partial \psi}{\partial x^{\bar{\rho}}}-e \psi^{*} \alpha_{\bar{\rho}} \psi \phi_{\bar{\rho}}+m c^{2} \psi^{*} \sigma \psi,
$$

i.e., the energy operator is

$$
H=\alpha_{\bar{\rho}}\left(\frac{h}{2 \pi i} \frac{\partial}{\partial x^{\bar{\rho}}}-\frac{e}{c} \phi_{\bar{\rho}}\right)+m c \sigma .
$$

Furthermore we have

$$
T_{4 \bar{\nu}}^{\prime}=\mathbf{R} \omega \psi^{*} \frac{\partial \psi}{\partial x^{\bar{\nu}}}-e \psi^{*} \psi \phi_{\bar{\nu}}+\mathbf{R} \frac{\omega}{4} \frac{\partial}{\partial x^{\bar{\rho}}}\left(\psi^{*} \alpha_{\bar{\nu}} \alpha_{\bar{\rho}} \psi\right)
$$

if we set

$$
\alpha_{1} \alpha_{2}=\mu_{3}
$$

and also cyclically, then we have for example

$$
T_{41}^{\prime}=\mathbf{R} \omega \psi^{*} \frac{\partial \psi}{\partial x^{1}}-e \psi^{*} \psi \phi_{1}+\frac{\omega}{4}\left\{\frac{\partial}{\partial x^{2}}\left(\psi^{*} \mu_{3} \psi\right)-\frac{\partial}{\partial x^{3}}\left(\psi^{*} \mu_{2} \psi\right)\right\} .
$$

The momentum operator is therefore:

$$
P_{\bar{\nu}}=\frac{h}{2 \pi i} \frac{\partial}{\partial x^{\bar{\nu}}}-\frac{e}{c} \phi_{\bar{\nu}}
$$

on the other hand we get for the angular momentum:

$$
\begin{gathered}
M_{1}=x^{2} T_{43}^{\prime}-x^{3} T_{42}^{\prime}=\mathbf{R} \omega \psi^{*}\left(x^{2} \frac{\partial}{\partial x^{3}}-x^{3} \frac{\partial}{\partial x^{2}}\right) \psi-c \psi^{*} \psi\left[x^{2} \phi_{3}-x^{3} \phi_{2}\right] \\
+\frac{\omega}{4}\left\{x^{2} \frac{\partial}{\partial x^{1}}\left(\psi^{*} \mu_{2} \psi\right)-x^{2} \frac{\partial}{\partial x^{2}}\left(\psi^{*} \mu_{1} \psi\right)-x^{3} \frac{\partial}{\partial x^{3}}\left(\psi^{*} \mu_{1} \psi\right)+x^{3} \frac{\partial}{\partial x^{1}}\left(\psi^{*} \mu_{3} \psi\right)\right\},
\end{gathered}
$$

and consequently for the corresponding operator

$$
M_{1}=\frac{h}{2 \pi i}\left(x^{2} \frac{\partial}{\partial x^{3}}-x^{3} \frac{\partial}{\partial x^{2}}\right)-\frac{e}{c}\left(x^{2} \phi_{3}-x^{3} \phi_{2}\right)+\frac{i \mu_{1}}{2} .
$$

2. In the preceeding we have assumed Bose-Einstein statistics for the tetrads $h_{i, \nu}$, i.e., we have chosen a minus sign in the bracket symbol for the CR's. One could ask whether it would be possible to apply Fermi statistics to the tetrads. The criterion for the admissibility of CR's with a plus sign is the following (cf. H.P. I, p. 29): the usual bracket symbols (with the minus $\operatorname{sign}$ ) $\left[\mathcal{G}_{\mu}, Q_{\alpha}\right],\left[\mathcal{G}_{\mu}, \mathcal{P}^{\alpha}\right]$ must

${ }^{25}$ Cf. also H. Tetrode, Ztschr f. Phys. 60, p. 858, 1928. Fomulae (13) and (16) as well as the text on p. 862 . 
assume the same value when one replaces the minus sign in $\left[Q_{\alpha}, Q_{\beta}\right],\left[\mathcal{P}^{\alpha}, \mathcal{P}^{\beta}\right]$, and $\left[Q_{\alpha}, \mathcal{P}^{\beta}\right]$ with a plus sign.

Applying this criterion the answer with reference to the tetrads is no, since one sees from the form of the Hamiltonian (quadratic in the $\mathcal{P}^{\alpha}$ ) that in the transition from a plus to a minus sign $\left[\overline{\mathcal{H}}_{0}, Q_{\alpha}\right]$ undergoes a change; in the bracket symbols belonging to the constituents of $\mathcal{H}_{0}$ the two terms quadratic in the $\mathcal{P}^{\alpha}$ are different, and the differences do not compensate each other.

3. The pure (vacuum) gravitational field could be described by the $g_{\mu \nu}$ instead of the $h_{i, \nu}$. Then we would be dealing with another variety of the "second case" and due to the general covariance group we would obtain four identities of the form $\left(\mathcal{P}^{\alpha}+\mathcal{R}^{\alpha 4}\right) c_{\alpha \nu}^{4}=0$.

\section{Summary}

1. When the Lagrangian function $\mathcal{L}\left(Q_{\alpha} ; \dot{Q}_{\alpha}\right)$ transforms under the group ${ }^{26}$

$$
\left\{\begin{array}{c}
\delta x^{\nu}=\alpha_{r}^{\nu, 0}(x) \xi^{r}(x), \\
\delta Q_{\alpha}=c_{\alpha r}^{0}(x, Q) \xi^{r}+c_{\alpha r}^{\sigma} \frac{\partial \xi^{r}}{\partial x^{\sigma}}
\end{array}\right.
$$

as a scalar density, then there hold identities between the $Q$ and the conjugate momenta $\mathcal{P}$ of the form

$$
\mathcal{F}_{r} \equiv \underline{\mathcal{P}^{\alpha} c_{\alpha r}^{4}}=0 .
$$

In case $\mathcal{L}+\mathcal{L}^{\prime}$, but not $\mathcal{L}$, is a scalar density, where $\mathcal{L}^{\prime}$ is linear in the second derivatives of the $Q_{\alpha}$, then $\mathcal{P}^{\alpha}+\mathcal{R}^{\alpha 4}$ appears everywhere in place of $\mathcal{P}^{\alpha}$.

2. Consequently the solution of the equations

$$
\mathcal{P}^{\alpha}=\frac{\partial \mathcal{L}}{\partial \dot{Q}_{\alpha}}
$$

for the $\dot{Q}_{\alpha}$ takes the form

$$
\dot{Q}_{\alpha}=\dot{Q}_{\alpha}^{0}(\mathcal{P}, Q)+\lambda^{r} c_{\alpha r}^{4},
$$

with arbitrary spacetime functions $\lambda^{r}$.

The Hamiltonian thereby takes the form

$$
\mathcal{H}=\mathcal{H}_{0}(\mathcal{P}, Q)+\lambda^{r} \mathcal{F}_{r}
$$

The basic equations of the theory are the canonical field equations, the canonical CR's, and the constraints

$$
\mathcal{F}_{r}=0 \text { and } \frac{d \mathcal{F}_{r}}{d x^{4}}=0
$$

3. The infinitesimal transformations of the group can be expressed as

$$
\begin{gathered}
\omega \delta^{*} \Phi=[\overline{\mathcal{M}}, \Phi] . \\
\mathcal{M}=\underline{\mathcal{P}^{\alpha} \delta Q_{\alpha}}-\mathcal{G}_{\mu} \delta x^{\mu} .
\end{gathered}
$$

\footnotetext{
${ }^{26}$ For the purpose of clarity we specialize the formulas to the physically important case $j=1$.
} 
( $\Phi$ is an arbitrary function that depends only on $Q$ and $\mathcal{P} ; \mathcal{G}_{\mu}$ is the (pseudo) energy-momentum density).

A special case of $\overline{\mathcal{M}}$ on an arbitrary slice $x^{4}=x_{0}^{4}$ is $\overline{\epsilon^{r} \mathcal{F}_{r}}$. It follows from $\mathcal{F}_{r}=0$ that the $\mathcal{F}_{r}$ commute amongst themselves, i.e., that the constraints $\mathcal{F}_{r}=0$ are compatible.

Furthermore, due to the field equations,

$$
\frac{d \overline{\mathcal{M}}}{d x^{4}}=0
$$

from which it follows that

$$
\overline{\mathcal{M}}=\int d x^{1} d x^{2} d x^{3}\left\{\mathcal{F}_{r} \frac{\partial \xi^{r}}{\partial x^{4}}-\frac{d \mathcal{F}_{r}}{d x^{4}} \xi^{r}\right\} .
$$

and because of the field equations (temporal evolution of the constraints)

$$
\frac{d^{2} \mathcal{F}_{r}}{\left(d x^{4}\right)^{2}} \equiv 0
$$

4. The basic system of equations is invariant under the group.

5. The electromagnetic field, the Dirac material field, and the gravitational field including all interactions were treated as examples. The relevant groups are the gauge invariance group, the true Bein covariance group, and the group of general relativity theory.

In particular, as regards gravitation, it is not possible to quantize the corresponding field quantities with Fermi statistics.

Acknowledgements. I express my sincere thanks to Prof. Pauli for his suggestion to undertake this work and for his valuable advice.

\section{Comments}

[1] An ellipsis is missing in these equations. They should read

$$
\left\{\begin{array}{c}
\delta x^{\nu}=a_{r}^{\nu, 0}(x) \xi^{r}(x)+a_{r}^{\nu, \sigma}(x) \frac{\partial \xi^{r}}{\partial x^{\sigma}}+\ldots+a_{r}^{\nu, \sigma \ldots \tau}(x) \frac{\partial^{k} \xi^{r}}{\partial x^{\sigma} \ldots \partial x^{\tau}}, \\
\delta Q_{\alpha}=c_{\alpha r}^{0}(x, Q) \xi^{r}(x)+c_{\alpha r}^{\sigma}(x, Q) \frac{\partial \xi^{r}}{\partial x^{\sigma}}+\ldots+c_{\alpha r}^{\sigma \ldots \tau}(x, Q) \frac{\partial^{j} \xi^{r}}{\partial x^{\sigma} \ldots \partial x^{\tau}} .
\end{array}\right.
$$

[2] Note that with these definitions Rosenfeld is accomodating the inclusion of transformations for which $\delta x^{\nu}=0$, in which case he is evidently taking $k=-1$. Thus the local Lorentz and $\mathrm{U}(1)$ transformations treated in Part 2 will also satisfy the condition

$$
j \geq k+1,
$$

where $k=-1$ and $j=0$. See equation (120).

[3] This is a generalization of the notion of contravariant that applies also to variations that are not generated by coordinate transformations. Thus as we shall see internal gauge transformations are included. When the $\alpha$ become coordinate tensor indices one recovers the usual coordinate definition.

[4] The commutator will not depend on either $\dot{Q}_{\alpha}$ or $\mathcal{P}^{\alpha}$.

[5] The reference is to the rewritten form of equation (21) that follows equation (29). 
[6] The line containing the commutators may be written as the nested commutator

$$
\frac{1}{2}\left[\mathcal{A}^{\alpha \beta^{\prime}},\left[\mathcal{P}^{\gamma^{\prime}}-\mathcal{D}^{\gamma^{\prime}}, \mathcal{A}_{\gamma^{\prime} \beta^{\prime}}\right]\right] .
$$

This expression vanishes by virtue of the second implication.

[7] This classical equality is established by Rosenfeld in Ann. de l'I.H.P. (1932), 25.

[8] If the spatial boundary is taken to be finite it appears to be sufficient for Rosenfeld to assume that the field quantities take the same constant value at each coordinate boundary. See the remark [9] preceding equation (44). On the other hand if Rosenfeld is contemplating a falloff behavior at spatial infinity he needs to assume that the Lagrangian asymptotically approaches zero. An alternative not mentioned by Rosenfeld would be to treat a spatially compact manifold.

[9] The correct expression would be $\left[\left[\overline{\mathcal{G}}_{\mu}, \overline{\mathcal{G}}_{\nu}\right], \Phi\right]=0$.

[10] These transformations played an important role much later in Dirac's Hamiltonian formulation of general relativity in 1958 where he found canonical variables that were invariant under these transformations. Bergmann called such variables D-invariant in recognition of Dirac's discovery. Such variables were however isolated in a different but related context by Paul Weiss in 1938 .

[11] More precisely,

$$
\left[\mathcal{F}_{s}\left(\boldsymbol{x}, x_{0}^{4}\right), \mathcal{F}_{r}\left(\boldsymbol{x}^{\prime}, x_{0}^{4}\right)\right]=\delta^{3}\left(\boldsymbol{x}-\boldsymbol{x}^{\prime}\right) c_{r s}^{t} \mathcal{F}_{t}\left(\boldsymbol{x}, x_{0}^{4}\right)
$$

Recall that Rosenfeld is confining himself here to transformations for which $\delta x^{\mu}=0$.

[12] Rosenfeld evidently assumes that the variations vanish on the spatial boundary.

[13] There is a puzzle here. As Rosenfeld notes, the requirement that arbitrary time derivatives of the constraints $\mathcal{F}_{r}=0$ vanish is an internal consistency requirement. It is independent of the analysis of the generator $\mathcal{M}$ that he has just undertaken. With this requirement in mind, the result that $\frac{d^{j+1} \mathcal{F}_{r}}{\left(d x^{4}\right)^{j+1}}=0$ may be viewed as a consistency check. What Rosenfeld has actually proven here is that on account of the requirement that $\frac{d^{i} \mathcal{F}_{r}}{\left(d x^{4}\right)^{2}}=0$, the generator $\mathcal{M}$ must vanish. But he never says this explicitly.

[14] We are to understand the presence of arbitrary functions as Rosenfeld's definition of "missing".

[15] We are still considering here the special case $j=1$.

[16] Keep in mind that in this definition $k+1 \leq i \leq j$, so these derivatives need not vanish.

[17] Rosenfeld is demonstrating here that the cumulative variation in $\Phi$ obtained by first performing an infinitesimal transformation generated by $\overline{\mathcal{N}}$ and then followed by a transformation generated by $\overline{\mathcal{M}}$ can be written as a transformation generated by $\overline{\mathcal{M}}$ followed by a transformation generated by an altered generator, namely $\overline{\mathcal{N}}+\frac{1}{\omega}[\overline{\mathcal{M}}, \overline{\mathcal{N}}]$ The transformed fields under the first transformation are labeled by a "prime", whereas the transformed fields under the second transformation are denoted with a "tilde". Thus

$$
\Phi^{\prime}=\Phi+\frac{1}{\omega}[\overline{\mathcal{N}}, \Phi],
$$

and to be unambiguous Rosenfeld should denote the second transformed field as $\widetilde{\left(\Phi^{\prime}\right)}$ and not $\left(\tilde{\Phi^{\prime}}\right)$, Thus

$$
\begin{aligned}
\widetilde{\left(\Phi^{\prime}\right)} & =\Phi^{\prime}+\frac{1}{\omega}\left[\overline{\mathcal{M}}, \Phi^{\prime}\right]=\Phi+\frac{1}{\omega}[\overline{\mathcal{N}}, \Phi]+\left[\overline{\mathcal{M}}, \Phi+\frac{1}{\omega}[\overline{\mathcal{N}}, \Phi]\right] \\
& =\Phi+\frac{1}{\omega}[\overline{\mathcal{N}}, \Phi]+\frac{1}{\omega}[\overline{\mathcal{M}}, \Phi]-\frac{1}{\omega^{2}}([\Phi,[\overline{\mathcal{N}}, \overline{\mathcal{M}}]]+[\overline{\mathcal{N}},[\Phi, \overline{\mathcal{M}}]]) \\
& =\Phi+\frac{1}{\omega}[\overline{\mathcal{M}}, \Phi]+\frac{1}{\omega}\left[\overline{\mathcal{N}}+\frac{1}{\omega}[\overline{\mathcal{M}}, \overline{\mathcal{N}}], \Phi+\frac{1}{\omega}[\overline{\mathcal{M}}, \Phi]\right] \\
& \left.=\tilde{\Phi}+\frac{1}{\omega}\left[\overline{\mathcal{N}}+\frac{1}{\omega}[\overline{\mathcal{M}}, \overline{\mathcal{N}}], \tilde{\Phi}\right]\right]
\end{aligned}
$$

where in the second line the Jacobi identity was used and in the third line we ignore terms of order $\left(\frac{1}{\omega}\right)^{3}$. Equivalently, he has computed the generator of the commutator 
of the two transformations generated by $\overline{\mathcal{N}}$ and $\overline{\mathcal{M}}$; this infinitesimal commutator is generated by $\frac{1}{\omega}[\overline{\mathcal{M}}, \overline{\mathcal{N}}]$.

[18] Rosenfeld does not employ the identity (81) to prove $\left(80^{\prime}\right)$. The key observation here is that the coefficients of $Q_{\alpha, \nu \rho}$ in $r_{r, \rho}^{\rho}$ must vanish, where $r_{r}^{\rho}:=r^{\alpha \rho} c_{\alpha r}$. But since

$$
r_{r, \rho}^{\rho}=\frac{\partial r_{r}^{\rho}}{\partial Q_{\alpha}} Q_{\alpha, \rho}+\frac{\partial r_{r}^{\rho}}{\partial Q_{\alpha, \nu}} Q_{\alpha, \nu \rho}
$$

the desired coefficient is

$$
\frac{\partial r_{r}^{\rho}}{\partial Q_{\alpha, \nu}}+\frac{\partial r_{r}^{\nu}}{\partial Q_{\alpha, \rho}}=0
$$

This is $\left(80^{\prime}\right)$.

[19] Rosenfeld does not say this explicitly in the text, but it does turn out that the particular solution $Q_{\alpha}^{0}$ is still given by (32), as we now show. The new identity is

$$
c_{\alpha r}\left(\mathcal{P}^{\alpha}+\mathcal{R}^{\alpha 4}\right) \equiv c_{\alpha r}\left(\mathcal{A}^{\alpha \beta} \dot{Q}_{\beta}+\mathcal{R}^{\alpha 4}+\mathcal{D}^{\alpha}\right) \equiv 0
$$

Then since $\mathcal{R}^{\alpha 4}$ and $\mathcal{D}^{\alpha}$ are independent of $\dot{Q}_{\beta}$, if follows that $c_{\alpha r}$ is still a null vector, i.e.

$$
c_{\alpha r} \mathcal{A}^{\alpha \beta} \equiv 0
$$

and furthermore

$$
c_{\alpha r}\left(\mathcal{R}^{\alpha 4}+\mathcal{D}^{\alpha}\right) \equiv 0
$$

Now as in case 1 we can use the null vector, labeled by $\gamma^{\prime \prime}, c_{\alpha^{\prime}}^{\prime \gamma^{\prime \prime}}=\mathcal{A}^{\gamma^{\prime \prime} \beta^{\prime}} \mathcal{A}_{\beta^{\prime} \alpha^{\prime}}$ and $c_{\alpha^{\prime \prime}}^{\prime \gamma^{\prime \prime}}=-\delta_{\alpha^{\prime \prime}}^{\gamma^{\prime \prime}}$. So we deduce that since

$$
c_{\alpha}^{\prime \gamma^{\prime \prime}}\left(\mathcal{P}^{\alpha}+\mathcal{R}^{\alpha 4}\right) \equiv 0
$$

and

$$
\begin{gathered}
c_{\alpha}^{\prime \gamma^{\prime \prime}}\left(\mathcal{R}^{\alpha 4}+\mathcal{D}^{\alpha}\right) \equiv 0, \\
c_{\alpha}^{\prime \gamma^{\prime \prime}} \mathcal{P}^{\alpha}=c_{\alpha^{\prime}}^{\prime \gamma^{\prime \prime}} \mathcal{D}^{\alpha},
\end{gathered}
$$

or

$$
\mathcal{P}^{\alpha^{\prime \prime}}-\mathcal{D}^{\alpha^{\prime \prime}}=\mathcal{A}^{\gamma^{\prime \prime} \beta^{\prime}} \mathcal{A}_{\beta^{\prime} \alpha^{\prime}}\left(\mathcal{P}^{\alpha^{\prime}}-\mathcal{D}^{\alpha^{\prime}}\right)
$$

[20] Recall (80).

[21] We have corrected an obvious typographical error in the second of equations (95).

[22] Note that in cgs units, the dimension of the four-potential $\phi_{\mu}$ is mass $^{1 / 2}$ length ${ }^{1 / 2}$ time $^{-1}$, and the dimension of the descriptor $\xi$ is therefore mass $^{1 / 2}$ length $^{3 / 2}$ time $^{-1}$. The factor $\frac{e}{\omega} \xi$ is therefore dimensionless.

[23] This specialization to the flat case should read

$$
h_{i, \nu}=e_{i} \delta_{i, \nu}
$$

as it is correctly expressed immediately following equation (128c).

[24] This claim appears to be false because one cannot ignore the generally time dependent terms at spatial infinity.

[25] Felix Klein was apparently the first to publish the analogue of the identity (56c), for Einstein's first order Lagrangian of 1916, though it is really an application of Noether's second theorem whose origins predate Klein's paper. Rosenfeld does not give an explicit proof that his expression is equivalent to the four non-dynamical Einstein equations. He seems to argue, rather, that since this is a vanishing expression that does not involve accelerations it must be equivalent.

[26] $\delta e_{I \nu}=\xi_{I J} e_{\nu}^{J}$.

[27] Rosenfeld apparently means by "locally invariant" what we would call today "covariant". 
[28] The Rosenfeld expression should actually be $\mathcal{T}_{i k}^{\prime \prime}=e_{k} \mathcal{T}_{i}{ }^{\nu} h_{k, \nu}$. Transcribing in modern notation this is

$$
\mathcal{T}^{\prime \prime I K}=\mathcal{T}^{I \mu} e_{\mu}^{K} .
$$

[29] The mass term appears with the wrong sign in Rosenfeld's matter Lagrangian. The corrected expression is therefore

$$
T^{\prime 00}=-i \hbar c \bar{\psi} \Gamma^{a}\left(\frac{\partial}{\partial x^{a}}-i \frac{e}{\hbar c} \phi_{a}\right) \psi+m c^{2} \bar{\psi} \psi
$$

